\title{
CIRCULACIÓN DE BIENES, USO DEL ESPACIO INTERIOR Y ESPACIOS PROGRAMADOS EN LOS ARCHIPIÉLAGOS DE FUEGO-PATAGONIA EN TIEMPOS RECIENTES
}

\author{
LUIS ALBERTO BORRERO ${ }^{a}$, FLAVIA MORELLO ${ }^{b} \&$ MANUEL SAN ROMÁN ${ }^{\mathrm{b}}$
}

\begin{abstract}
RESUMEN
A partir de consideraciones acerca de la geografía cultural para el archipiélago fueguino, se delibera sobre los registros de circulación de bienes, incluyendo la roca Miraflores, hematita, pirita y otros. Además, se discute su relación con la organización e instalaciones humanas que involucran el uso del hinterland o zonas interiores -casos como la búsqueda de corteza, caza de guanacos (Lama guanicoe) y huemul (Hippocamelus bisulcus), disposición de los muertos- y el equipamiento de individuos, canoas y lugares, considerando por ejemplo los corrales de pesca, varaderos y porteos o pasos de indio. Todos estos casos muestran la amplitud efectiva del nicho ecocultural para tiempos recientes en los archipiélagos de Fuego-Patagonia.
\end{abstract}

PALABRAS CLAVE: fueguino, Patagonia, arqueología, interacción, circulación, equipamiento, cazadores-recolectores.

\section{CIRCULATION OF GOODS, USE OF THE HINTERLAND AND PROGRAMMED SPACES IN THE ARCHIPELAGOS OF FUEGO-PATAGONIA DURING RECENT TIMES}

\begin{abstract}
On the basis of the concept of cultural geography of the fuegian archipelago a discussion of the circulation of goods, as Miraflores rock, hematite, pirite, and others is presented. In addition, its relationship to human organization and installations involving the use of the hinterland or interior zones -cases like bark search, guanaco (Lama guanicoe) and huemul (Hippocamelus bisulcus) hunting, disposition of the dead- and the equipment of individuals, canoes and places, considering for example fishing weirs, canoe dry docks and Indian passages, are discussed. All this evidence indicates the effective amplitude of the ecocultural niche during recent times in the Fuego-Patagonian archipelagos.
\end{abstract}

a Universidad de Buenos Aires e Instituto Multidisciplinario de Historia y Ciencias Humanas (IMHICIHU-CONICET), Saavedra 15, piso $5^{\circ}$ (1083 ACA), Ciudad Autónoma de Buenos Aires, Argentina. laborrero2003@yahoo.com

b Centro de Estudios del Hombre Austral, Instituto de la Patagonia, Universidad de Magallanes, Av. Bulnes 01890, Punta

Arenas, Chile. flavia.morello@umag.cl, manuel.sanroman@umag.cl 
KEY WORDS: Fuegian, Patagonia, archaeology, interaction, circulation, equipment, huntergatherers.

\section{INTRODUCCIÓN}

Puede defenderse que la circulación de bienes y las formas de uso del espacio -incluyendo elementos de equipamiento- atestiguan acerca de características de la geografía cultural de una región. Haremos tal evaluación para el archipiélago fueguino en forma independiente de la definición y extensión espacial de las entidades étnicas históricamente conocidas, debido en parte a que utilizaremos un marco temporal un poco más amplio, incluyendo así unidades para las que sólo existen definiciones arqueológicas. Por otra parte, también nos sustentamos en que este período más reciente, que abarca aproximadamente los últimos mil años, en general estuvo sujeto a una dinámica cultural de tal intensidad como para que el potencial de cambio dentro de las caracterizaciones culturales sea altísimo. Vemos esta entrada a la discusión de tiempos tanto arqueológicos como etnográficos como una atractiva alternativa a otros enfoques espacialmente más restringidos.

\section{CIRCULACIÓN DE BIENES}

Son escasos los hallazgos arqueológicos que se refieren en forma no ambigua a recursos lejanos, los que por cierto han sido reconocidos como poco abundantes (Orquera \& Piana, 1995, p. 27). Muchas veces en la literatura histórica se exhibe el intercambio como la causa de los desplazamientos a través de los pasos, pero existen muy pocas evidencias al respecto. El uso de pasos u otras vías de comunicación tiene mucho más sentido simplemente como parte de los desplazamientos de sociedades muy móviles, incluyendo sus requerimientos de interacción social (por ejemplo, visitas) y conectividad. En el registro etnohistórico los intercambios y las visitas son parte integral, por ejemplo, en los casos de las misiones del Cabo de Hornos (Martial, 2007 [1891]) y

\footnotetext{
Existen también referencias a fuentes más alejadas. Gusinde (1991a [1974], pp. 186-187) habla de la isla Solitaria en el golfo de Penas (ver Emperaire, 1963 [1955], pp. 112, 120) y citando a Giglioli menciona Puerto Edén. Fitz Roy (2016 [1839], p. 175) menciona la existencia de pirita cerca del
}

anglicanas (Bridges, 1951), pero también existen registros muy anteriores (ver abajo). Discutiremos brevemente algunos bienes que circulaban lejos de sus lugares de obtención.

\section{Pirita}

La fuente de pirita -mineral utilizado para hacer fuego por percusión- más referida en la literatura es la del seno Mercurio, isla Capitán Aracena (Fig. 1) (Martial, 2007 [1891], p. 41; Chapman, 2010, pp. 42, 644; Gibbons \& Harambour, 2016; Gallardo et al. 2018), muchas veces referida como isla Clarence (Cooper, 1917, pp. 191-192; Bridges, 1951, p. 64; Orquera \& Piana, 2015, p. 361). Se ha indicado que era "a region where Yahgan and Alacaloof mixed" (Bridges, 1951, p. 64), aunque no hay evidencias de que hayan convergido allí.

Otras fuentes de pirita han sido mencionadas a lo largo y a lo ancho de los archipiélagos. Lothrop habla de pirita en la isla Merton (1928, pp. 65, 130) (para algunos ha querido decir Morton, Chapman, 2010, p. 177; Orquera \& Piana, 2015, p. 361, lo que quiere decir el segmento occidental del canal Beagle), bahía Unión y lago Fagnano (Lothrop, 1928, p. 65]. También han sido mencionadas las islas Skyring y Fury cerca de la península Brecknock, no lejos del seno Mercurio (Hyades \& Deniker, 2007 [1891], pp. 91-92; Chapman, 2010, p. 115). Más al norte Alberto Achacaz Walakial, kawésqar, menciona "que esas piedras las encontraban de Puerto Natales hacia afuera y también en la isla Clarence /Capitán Aracena/" (Vega Delgado, 1995, p. 22). Hyades menciona pirita en un islote cercano a la isla Packsaddle en bahía Nassau ${ }^{1}$, mientras que Fitz Roy halló pirita en la isla Gilbert oriental, entre Stewart y Londonderry (Chapman, 2010, p. 115). Entonces, tal vez el seno Mercurio sea la fuente para la cual nos ha llegado información etnográfica específica, lo cual

golfo de Trinidad. Francisco Arroyo, kawésqar de Puerto Edén, informa de una isla de la costa sur del golfo de Penas como fuente de pedernal para hacer fuego (com. pers., 2009). 


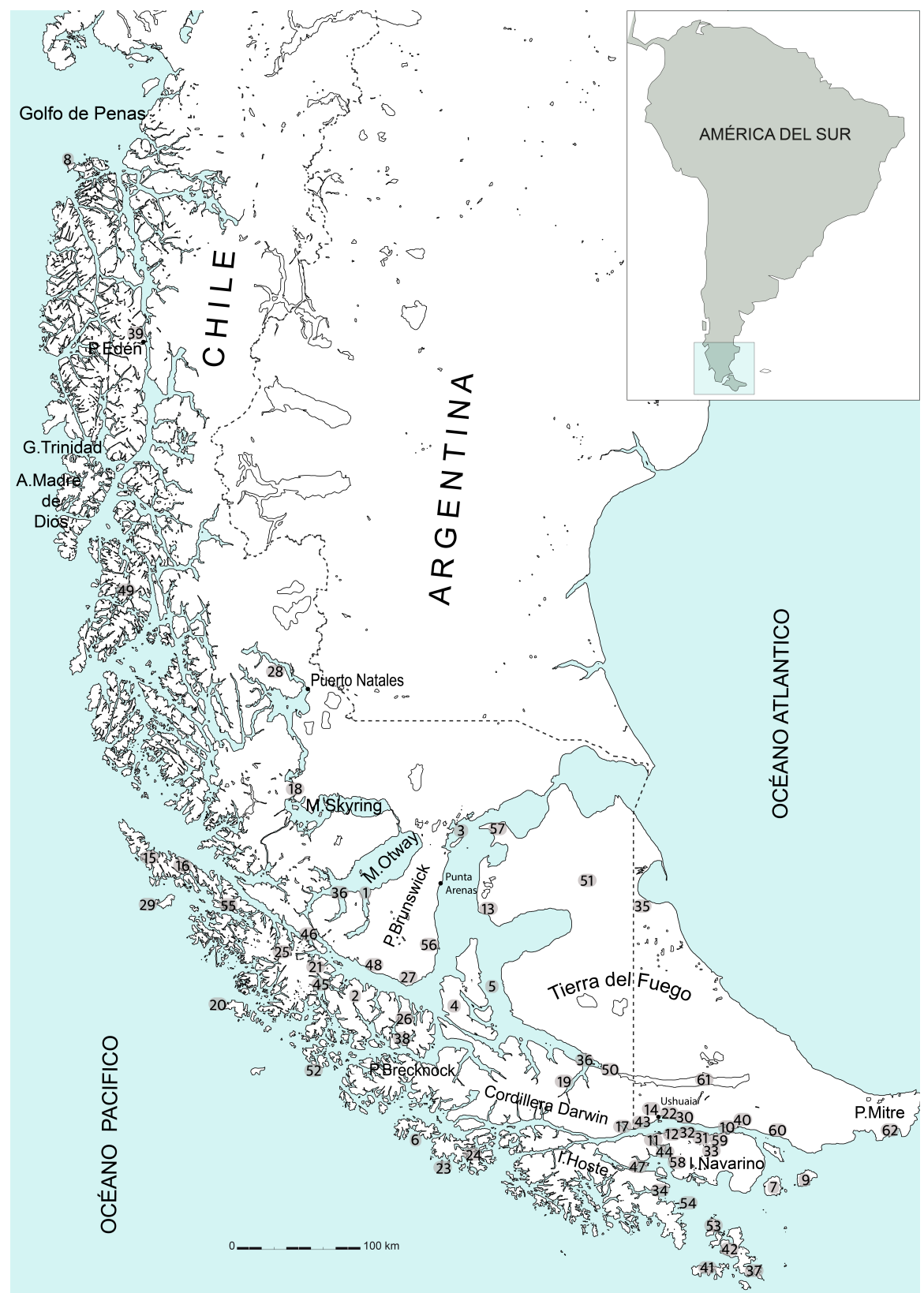

Fig. 1. Ubicación de localidades y sitios arqueológicos de Patagonia y Tierra del Fuego. (1) fiordo Silva Palma, (2) isla Clarence, (3) isla Isabel, (4) isla Dawson, (5) canal Whiteside, (6) isla Gilbert, (7) isla Lennox, (8) isla Solitaria, (9) isla Nueva, (10) isla Gable, (11) canal Murray, (12) isla Cole, (13) cabo Monmouth, (14) valle Andorra, (15) isla Desolación, (16) Puerto Churruca, (17) Yendegaia/Rockshelter 3, (18) seno Excelsior, (19) fiordo Parry, (20) cabo Gloucester, (21) bahía Choiseul, (22) sitios 409/ Río Pipo 17/ Ajej I, (23) isla Alikhoolip, (24) isla Londonderry, (25) isla Santa Inés, (26) isla Capitán Aracena, (27) cabo Froward, (28) sitio Cueva de los Niños, (29) isla Recalada, (30) sitios Mischihuen III/Shamakush Playa Larga/Lancha Packewaia/ Túnel I-II-VII-XII, (31) bahía Mejillones, (32) bahía Unión, (33) sitios Cerro Bandera 1 y Paso Laguna del Salto 2, (34) bahía Tekenika, (35) sitio Mandíbulas, (36) isla Tres Mogotes, (37) isla Herschel, (38) seno Mercurio, (39) sitio Jekchal, (40) sitio Imiwaia 1, (41) isla Hermite, (42) islas Wollaston, (43) Lapataia/isla El Salmón, (44) bahía Wulaia, (45) canal Bárbara, (46) cabo Quod, (47) isla Sapinj, (48) bahía Wood, (49) isla Hanover, (50) río Azopardo, (51) Chorrillo Miraflores, (52) isla Fury, (53) isla Grévy, (54) isla Packsaddle, (55) canal Abra, (56) sitios Punta Santa Ana 1-3, (57) cabo San Vicente, (58) Rockshelter 1, (59) ensenada Villarino, (60) sitios Heshkaia 34 y 35, (61) lago Fagnano, (62) bahía Valentín [Mapa elaborado por V. Sierpe y F. Morello]. 
no debe significar que es la única, la que se utilizó siempre o siquiera la principal. Resumiendo bien la cuestión, Ocampo y Rivas dicen que la pirita "estaría disponible en varios lugares del sur" (1996, p. 127) y debe recordarse que al menos una de las piritas de la isla Isabel no parece provenir de la isla Capitán Aracena (Gallardo et al. 2018).

Son muyescasas las referencias arqueológicas sobre estos materiales. Un fragmento de pirita fue recuperado en el componente reciente (Capa D) de Lancha Packewaia (Orquera \& Piana, 19931994) datado en unos 1.000 años AP (Orquera et al. 1977, p. 74), a lo que se suman hallazgos de piritas sin contexto cronológico en la isla Isabel (Gallardo et al. 2018) y en la localidad Mandíbulas en el norte de la Isla Grande (Karen Borrazzo, com. pers., 2014).

Piedras abrasivas, pómez, Miraflores y arenisca

Se trata de rocas utilizadas principalmente para trabajar el hueso. Se ha mencionado el uso de piedra pómez para pulir hueso o astiles, la que se encuentra en el canal Beagle en general (Lothrop, 1928, p. 143; Gusinde, 1986 [1937], p. 480) y no como escribió Lovisato sólo en las islas Nueva y Lennox (Orquera \& Piana, 2015, p. 330). Existe un registro de "pumice" procedente de la costa de la isla Gable (BM. 1933.240(1)) en la colección de mineralogía del Natural History Museum de Londres, pero pareciera un hallazgo aislado. Por otro lado, se destaca la playa de la isla Cole (bahía Santa Rosa, costa norte de la isla Navarino) en donde Denis Chevallay registró una playa exclusivamente compuesta de esta piedra pómez (com. pers., 2020).

También es posible que otras rocas sirvieran a estos propósitos, como por ejemplo tufas -cuyo origen se vincula a los lagos proglaciares de finales del Pleistoceno- que se han registrado en cabo Monmouth (Tierra del Fuego). Igualmente, otras piedras blandas como areniscas o rocas sedimentarias de grano fino habrian cumplido una función similar (Christensen, 2016), por ejemplo, en el contexto del sitio arqueológico Punta Santa Ana 3, estrecho de Magallanes (Morello, 2016).

Asimismo, Gusinde consigna el uso de arenisca para preparar arpones (1986 [1937], pp. $455,456)$ y un afilador de arenisca estaba incluido entre los objetos que portaba en una bolsa Capelo, haush (Outes, 1906).

La toba riolítica y la toba silicificada Miraflores -rocas con procedencia en el extremo norte de la Isla Grande (Borrazzo, 2012)- tienen una distribución prácticamente panfueguina. Sin embargo, en el archipiélago fueguino austral y al sur de la cordillera Darwin, la primera sólo está presente en la península Mitre (Pallo \& Borrazzo, 2016; Borrazzo et al. 2019). Esta toba riolítica Miraflores es adecuada para ser usada para la confección de alisadores o afiladores. Al respecto, Pallo y Borrazzo (2016) analizaron rutas para explicar la presencia de rocas Miraflores en lugares distantes a la fuente, como bahía Valentín. Para la circulación a través de la cordillera consideran más probable el uso de una ruta iniciada en las cabeceras del lago Fagnano en lugar de la más larga y compleja ruta vía el nacimiento del río Azopardo (i.e. Skottsberg, 1913). Más recientemente, Borrazzo y colaboradores (2019) discutieron los mecanismos de circulación. La procedencia puntual de estas rocas Miraflores agrega un elemento de evaluación de la conectividad con los ambientes del norte de la Isla Grande (Borrazzo, 2012).

\section{Piedras horadadas}

Se trata de artefactos de costosa confección cuyo propósito no es conocido. Se sabe de dos piedras horadadas recogidas por Lovisato y por Furlong (Cooper, 1917, pp. 213-214). Son piezas similares a la publicada por Borrero y Borrazzo para el suroeste de Santa Cruz (2011, p. 23; ver las descritas por Outes, 1905, pp. 432-438, también de Santa Cruz). Mansur-Franchomme et al. (1987-1988, p. 172) informan acerca de varios casos semejantes registrados en la Isla Grande y en Navarino, incluyendo hallazgos arqueológicos en un nivel antiguo de Túnel 1 (Orquera et al. 2012, p. 58) y en Túnel 12, lo que los lleva a sugerir un posible origen local, entendiéndose éste como fueguino. Más allá de que la única referencia cronológica excede el marco de este trabajo, la forma de adquisición de algunas de las escasas piezas documentadas, junto a sus características excepcionales, sirven para considerar la posibilidad de su mantenimiento en el sistema en el largo plazo a través de ciclaje lateral u otros procesos (Borrero \& Borrazzo, 2016). 


\section{Hematita y otros pigmentos}

Abundan las referencias al transporte de colorantes, utilizando estómagos de animales (Koppers, 1924, Figs. 25 y 28) o "the dried esophagus of a sea-lion" (Skottsberg, 1913, p. 603). Cooper menciona las bolsas para tierra roja kawésqar descritas por Sarmiento de Gamboa en los alrededores de la isla Hanover (1917, p. 205). Se conocen numerosos ejemplos en museos, como una bolsa con colorantes kawésqar hecha con intestinos de ballena depositada en el Museo Salesiano de Punta Arenas (Anónimo, 1992, p. 142) o los viales conteniendo "red paint" de Tierra del Fuego, "From C. Darwin. J. J. Henslow" (registro $N^{\circ} 1920$ ) y "white paint" (registro $\mathrm{N}^{\circ} 1921$ ) almacenados en el British Museum (obs. pers.).

Todas estas referencias pueden ser vistas, lógicamente, dentro del contexto principal de la pintura corporal (Fiore, 2005) o de la preparación de inhumaciones (Prieto \& Cárdenas, 2001; San Román \& Morello, 2001). Sin duda son también relevantes para las recientemente descubiertas pinturas rupestres en el Alero Martín González Calderón, en la isla Sapinj, en el seno Ponsomby al sur de la isla Hoste (González Calderón et al. 2014); en el Alero Picton 1 en la isla Picton (Muñoz et al. 2016) y en Yendegaia ${ }^{2}$ (BioBio Chile, 29 de diciembre de 2017). Sin duda estos colorantes también debieron ser utilizados para pintar artefactos muebles.

El análisis de pigmentos recuperados en sitios costeros, como Imiwaia I (ca. 154 años AP), realizado con un cuidadoso control tafonómico (Fiore et al. 2008), no presentó registros de "preparación" de los mismos. Efectivamente, los análisis indicaron ausencia de un ligamento orgánico, el que sí está presente en muestras fechadas ca. 940 años AP y antes (Dánae Fiore, com. pers., 2017). El trabajo de Fiore y colaboradores también evaluó lugares de disponibilidad natural de pigmentos. No hay evidencias de que esas fueran las fuentes utilizadas, aunque el tratamiento de las muestras podría enmascarar su origen. En todo caso, más de una fuente parece estar implicada y los autores aprendieron que éstas "were not ubiquitous, thus implying a special selection of pigments among all those available in the landscape" (Fiore et al.
2008, p. 3055), lo que nuevamente remite a una forma de organización pauteada del espacio bajo uso.

El registro de piedras con colorantes es descrito de manera llamativa por Junius Bird en dos menciones de su cuaderno de campo correspondientes a los años 1932-1933, durante su estadía en el canal Beagle. Primero, el día 13 de marzo de 1933 a propósito del hallazgo de un artefacto lítico -cuya descripción concuerda con un sobador- con colorante rojo encontrado en sus excavaciones en Puerto Pescado (costa norte de la isla Navarino): "found 2 flat pieces of stone, worn smooth on one side, on which red paint had been ground up or pulverized. Near one of these found one of the 'Grease' stones covered with red, on same level, a few inches further found a blank or reject also pure red. Later on 2 more water worn stones also with red. Indian Sarmiento came about noon and on seeing the painted stones said 'for punishing a criminal'. Said that it used to be the custom when it was decided that some criminal was to be punished they painted the family 'grease' stones red and hove them at the guilty party" (Bird, 1932, p. 167).

El segundo caso, apuntado en su viaje de regreso el 7 de abril de 1933, menciona haber revisado las notas del padre Thomas Bridges y consigna: "All notes by the late Rev. Thomas Bridges containing references [...] Contains account of a revenge expedition of a party of Yaghans to Ushuaia in which he says the spokesman carried 'white' stones ...nothing about painting stones red" (Bird, 1932, p. 178). El mismo evento es reproducido por Lucas Bridges a partir de las notas de su padre, que menciona "traía una piedra blanca en cada mano" (Bridges, 1952, p. 75).

\section{USO DEL HINTERLAND-INTERIOR}

Consideramos que, en grados variables, el espacio interior era importante dentro de la adaptación canoera. El sustento básico para esto es que ofrecía recursos críticos.

Más allá de que se trata de que el mundo canoero es por definición marítimo, con la mayoría de las actividades enfocadas en recursos acuáticos,

2 http://www.biobiochile.cl/noticias/artes-y-cultura/actualidad-cultural/...2/29/descubren-inedito-sitio-de-arte-rupestre-en-tierra-del-fuego.shtm 
existen evidencias de uso del interior. Lógicamente, ante la abrumadora evidencia de subsistencia marítima (Orquera \& Piana, 2015), el uso del interior está apenas mencionado (Prieto et al. 2000 , p. 90) y poco desarrollado en la literatura, situación que recientemente comenzó a cambiar (Álvarez et al. 2010; Fernández et al. 2018). Sin duda, son mayoría las referencias etnográficas clásicas, como la de Achacaz Walakial, "no nos gustaba movernos muy adentro en los bosques" (Vega Delgado, 1995, pp. 90). Sin embargo, exploraremos algunas alternativas conocidas de uso del interior.

Dijimos que la información etnográfica acerca de canoeros, por razones fácilmente comprensibles, no ha dado un lugar importante al interior. Chapman (2010, pp. 247ss) habla de las dificultades para la circulación terrestre, mientras que Gusinde destaca que "nadie atraviesa solo largas distancias en el bosque" (1986 [1937], p. 424). La imagen arqueológica ha estado, más allá de los esfuerzos de los arqueólogos para evitar proyectar los datos etnográficos, decididamente concentrada en la costa ${ }^{3}$, situación que sólo recientemente ha comenzado a cambiar. La exploración que aquí ofrecemos del uso del "interior" no busca cambiar la forma de considerar las adaptaciones humanas del sur de los archipiélagos, sino expandirla un poco. En otras palabras, explorar aquellos aspectos laterales que, aunque fueran mencionados y tal vez no siempre fueran cuantitativamente importantes, completan nuestra comprensión del modo de vida de uno o más sistemas adaptativos funcionando dentro de una cambiante geografía cultural (Borrero, 1991).

\section{Adquisición de rocas}

En el canal Beagle se ha reconocido una tendencia a colectar rocas locales en playas o afloramientos (Terradas, 2001), lo que confirma rangos de acción cortos (Breivik et al. 2016). Hay presencia ocasional de obsidiana verde, de procedencia en la zona del mar de Otway (Orquera \& Piana, 1999a) y restringida a un lapso temprano. Las fuentes etnográficas incluyen una referencia del diario de Thomas Bridges, del 21 de enero de 1884 acerca de un desplazamiento "por bote a Nagyoalacir [...] desde donde salimos hacia el norte al valle que lleva a Anawaia", un valle "renombrado en la tierra Yahgan como fuente de aprovisionamiento de pedernal o ágata para puntas de flecha y de lanza" (transcrito por Piana \& Martinioni, 1998, p. 2), que identifican con el valle de Andorra (ver también Orquera \& Piana, 2015, p. 370).

Por otra parte, existen evidencias arqueológicas, como las de procesamiento de rocas en el sitio Taller Altos del Varela I (Álvarez et al. 2010), en la cima del cerro Petersen (600$700 \mathrm{~m}$, a $10 \mathrm{~km}$ línea recta del canal Beagle). La localización del material por debajo del suelo sugiere una cronología reciente y la presencia de aristas frescas en material de superficie indica exposición reciente. Se trata de rocas de la Fm Lemaire -las más usadas en la regiónaunque el sitio no está espacialmente cercano a afloramientos. De hecho, estas rocas se pueden encontrar más cerca de la costa, por lo que probablemente la razón del acceso es otra, para Álvarez y colaboradores es la caza de guanacos. Por ello podría constituir un caso arqueológico de adquisición embedded, precisamente los que John Speth considera poco usuales (2018).

También debe mencionarse el Sitio 409, en el Parque Nacional Tierra del Fuego, Monte Martial, donde se produjo el hallazgo de lascas secundarias muy patinadas cercanas a unas lagunas de altura, al fondo del valle de Andorra, lugar que identifican con Anawaia (Piana \& Martinioni, 1998). Se trata del mismo valle "Aniawaia" de donde procedían "especially fine flints" (Lothrop, 1928, p. 130).

Finalmente se agregan los hallazgos realizados en dos sitios del interior de la isla Navarino, las canteras-taller Cerro Bandera 1 y Paso Laguna del Salto 2, ambos a más de $6 \mathrm{~km}$ de la costa en línea recta, al menos un día de caminata (Morello, 2018), muy significativos, pues allí no existen alternativas a los canoeros. Aquí se identificaron concentraciones de restos de talla en superficie en un contexto que sugiere funciones de cantera-taller y se asocia con la Fm Yaghan en el sector oriental de Dientes de Navarino (SERNAGEOMIN 2003) (Fig. 2). 

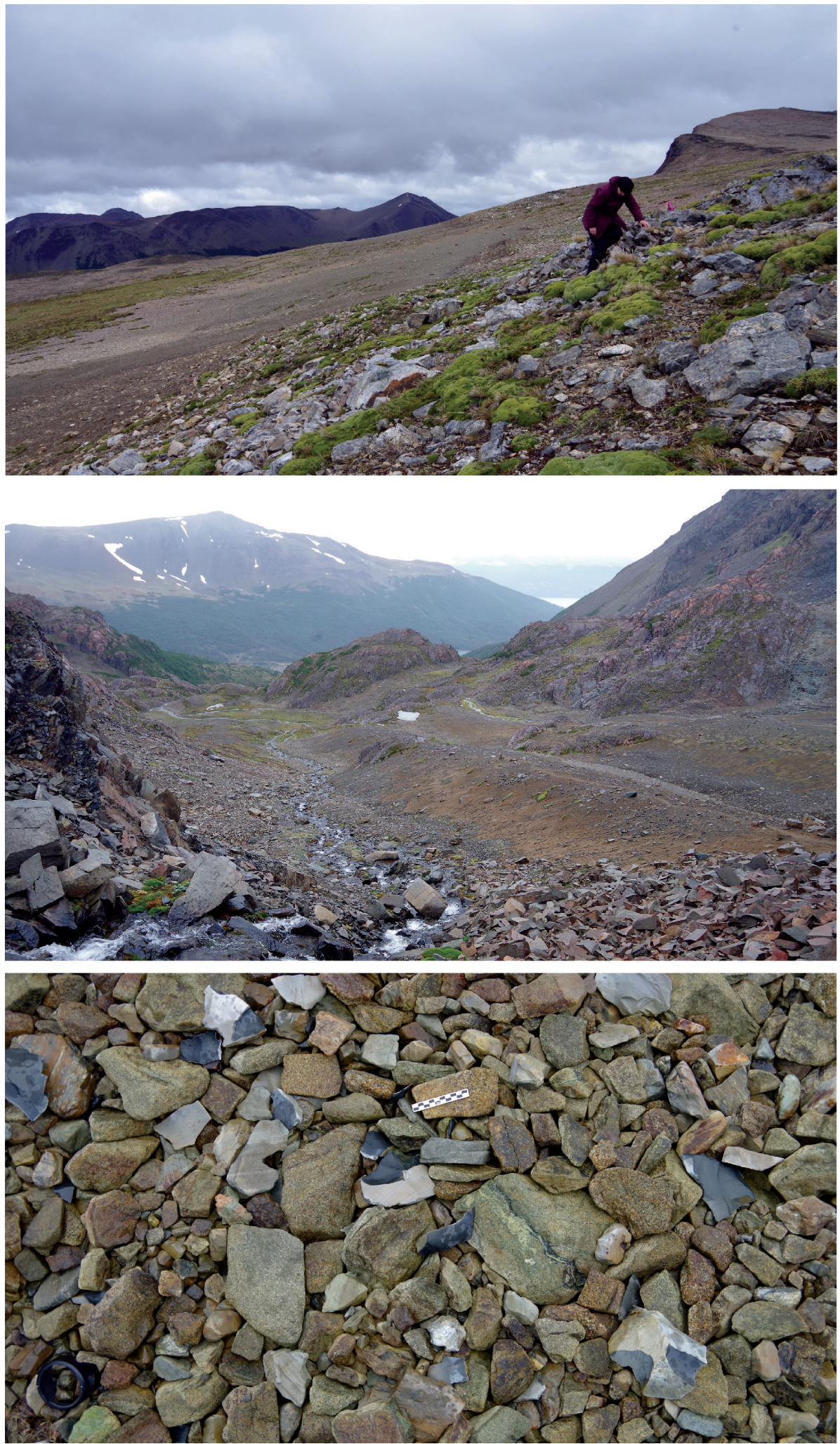

Fig. 2. Sitios arqueológicos del interior de isla Navarino. Superior: Cerro Bandera 1; Medio e Inferior: vista general y detalle de concentración lítica en Paso Laguna del Salto 2. Fotografías de Ismael Martínez. 


\section{Búsqueda de corteza}

La buena corteza para canoas debía buscarse en el interior del bosque, único lugar donde se encontraba "un trozo de corteza liso, uniforme y de suficiente longitud" (Gusinde, 1986 [1937], p. 424; Prieto et al. 2000, p. 90). Gusinde, citando a Despard, habla de recorrer distancias de 6.4-8 kilómetros (ib.), mientras que Hyades y Deniker (2007 [1891], p. 159) mencionan el recorrido de distancias de 8-10 km para casos de búsqueda de corteza.

\section{Porteos}

Se trata de sectores terrestres preparados para circular transportando las canoas (Fig. 3). Son llamados "arrastraderos" por Gabriela Paterito Caac, kawésqar (Aguilera \& Tonko, 2013, p. 221), y en algunos casos pueden incluir troncos a la manera de una sweet track o una corduroy road (Coles et al. 1973). Los porteos se plantean ante la existencia de barreras terrestres en circuitos marinos (Cooper, 1917; Chapman, 2010, pp. 114-117, 679). Por ejemplo, Martial describe en 1882-1883 “...istmo bajo i angosto que los indijenas atraviesan con sus piraguas para ir de Tequenica al seno Año Nuevo" (2007 [1891], p. 22). Sin duda los porteos son incursiones en el interior, con la posibilidad de que en algunos casos se circulara sin transportar canoas. Las razones para su uso son geográficas y tienen sentido en términos de esfuerzo, evitación de peligros y tiempo. Las más clásicas referencias son para los archipiélagos del norte (Byron, 1996 [1768], p. 89; Campbell, 2012 [1747]) o la identificación realizada por Katherine Routledge en 1913 de un paso de indios en Puerto Churruca, en la isla Desolación (Martinic, 2011, p. 59). Abundan las referencias de viajeros y etnógrafos sobre estos porteos (Martial, 2007 [1891], p. 22; Chapman, 2010, p. 679). La información lingüística es un registro claro de la importancia de este equipamiento de lugares y de la recurrencia de la acción de movilidad con porteos. Orquera y Piana (1999b, p. 260) citando a Thomas Bridges informan que "El lugar donde se había porteado una canoa para atravesar un istmo se llamaba töhtushana [...] la acción de cruzarlo llevando una canoa se decía tatushtushana". La información etnográfica muestra el caso de unas mujeres yagán que recorren un "porteo" a pie en la nieve, llevando niños, moluscos y antorchas; luego localizan sus canoas y reman hasta arribar a una

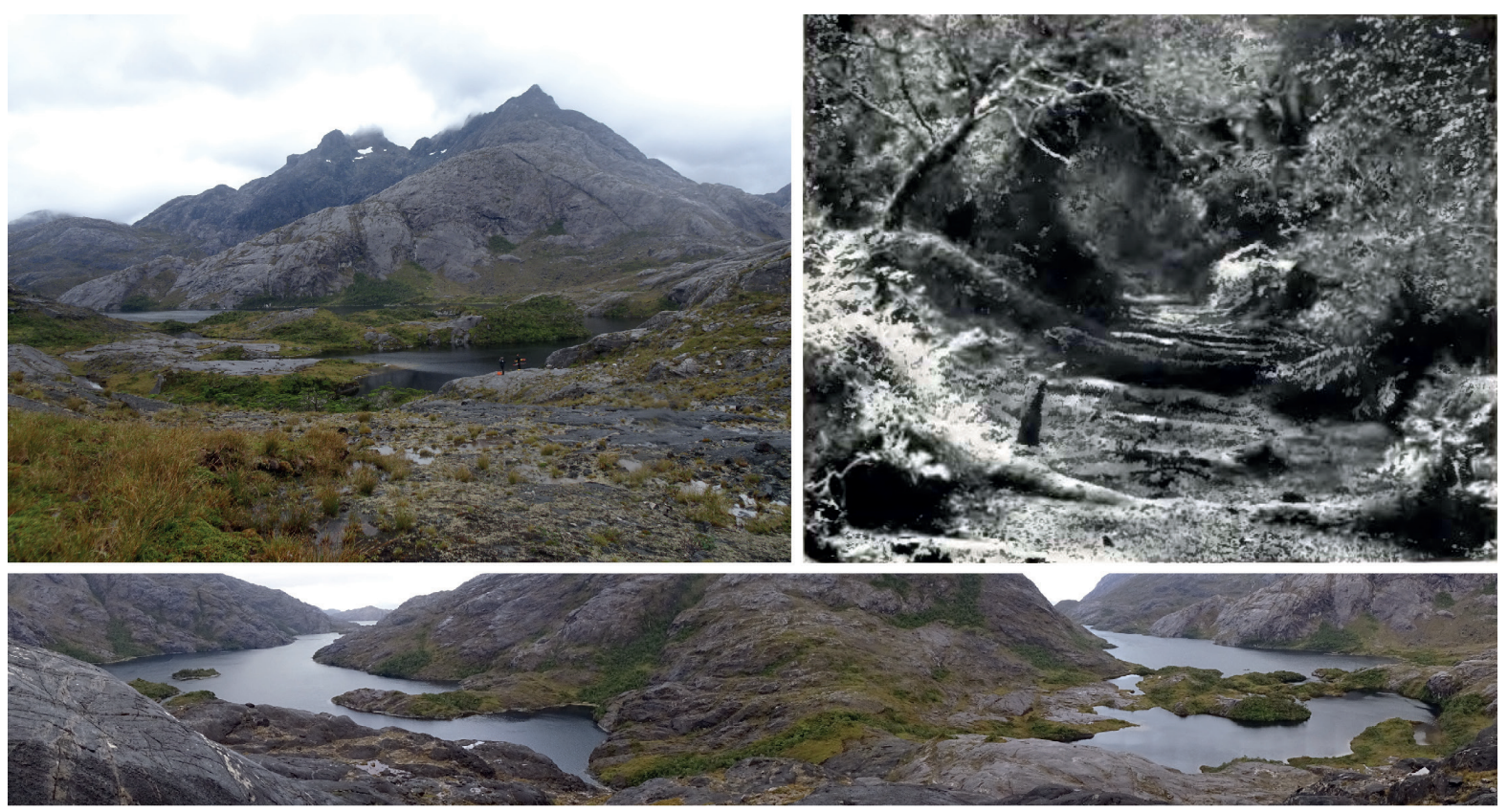

Fig. 3. Foto superior izquierda e inferior corresponden al paso de indios del seno Aragay, península Brecknock (fotografías de Javiera Mardones, 2020). Arriba a la derecha fotografía del porteo en Puerto Churruca, isla Desolación (modificado de Routledge, 1919, Fig. 14). 
ballena varada en el fiordo Doze en el seno Año Nuevo (Chapman, 2010, p. 574). En algunos casos se han llegado a desarmar canoas de tablas para afrontar el porteo (Martial, 2007 [1891], p. 33).

Maximiano Castillejo quien marca varias localizaciones en la cartografía que relaciona con porteos, sugiere que han de ser más que rutas o atajos (2016), sin ofrecer sustento para tales alternativas. Seguramente tenían otras funciones asociadas, pero ante todo son rutas y atajos que tienen pleno sentido en la geografía archipelágica. Cualesquiera sean los bienes asociados, sean chozas o canoas, no existe evidencia que los separe de su servicio a la función mencionada de circulación.

Para Prieto y colaboradores (2000) el uso de porteos representa una etapa de exploración, o sea, una de las primeras incursiones humanas en la zona. Esto es poco defendible, pues la localización y la construcción de los porteos implican manejo previo de la región, incluyendo gran conocimiento geográfico. La costosa inversión ingenieril de la construcción y limpieza de porteos se asocia con regularidad de paso y necesidad, no con improvisación ante lo desconocido. Además, se trata de lugares equipados -troncos, chozas, canoas, varaderos-, lo que también remite a fases de ocupación efectiva del espacio. Adicionalmente, como parte de su argumento de los porteos correspondiendo a una etapa de exploración, señalan que así se explicarían las Zonas Vacías marcadas por Laming-Emperaire para Última Esperanza (Laming-Emperaire, 1972, en Prieto et al. 2000). Sin embargo, la existencia de pasos asociados con escasas evidencias arqueológicas -por ejemplo, algunas pinturas o mínimas ocupacionesque unen espacios con ocupaciones fechadas en unos 4.500 años o más (Madre de Dios al oeste y Cerro Benítez al este), no podría explicarse durante todo ese tiempo como "exploración", sino más bien como lugares de circulación en tiempos de plena consolidación (Borrero, 1985).

Las dificultades de localización de los porteos están ejemplificadas por Skottsberg, quien visitó el seno Excelsior en el mar de Skyring y registró la presencia de un bosque impenetrable, en el que no se veía el sendero del paso de indios. Tras una búsqueda terrestre lo encontró y recorrió unos 400 $\mathrm{m}$ hacia el interior, hasta hallar dos lagunas, ambas alineadas con el porteo (Skottsberg, 1913, p. 589).
Aquí se diferencian claramente las dificultades de quien no tiene el conocimiento geográfico y ambiental, en relación con quienes lo poseen. Recorridos recientes de exploradores avezados han mostrado que la circulación por algunos de estos porteos, como el del fondo del fiordo Silva Palma, puede ser dificultosa pero realizable (Bruchhausen, 1966; Johnson, 1976). En otras palabras, ninguno de los porteos responde a la improvisación asociada con una etapa de exploración.

\section{Pasos que no implican transporte de la canoa}

Se trata de pasos que están mencionados como porteos, pero tienen características muy diferentes. El más destacado es el que va del fiordo Parry a Lapataia o Yendegaia (Prieto et al. 2000, pp. 87, 89; García, 2012; Niemeyer, 2013). Importa el hallazgo de armazones de chozas en el camino que une la bahía Blanca, en el fiordo Parry de la costa sur del seno del Almirantazgo, con el canal Beagle (Chevallay, 1999; García \& Prieto, 2017). También se ha mencionado el paso del seno del Almirantazgo al lago Fagnano (Furlong, 1917, p. 175; Chevallay, 1999; García \& Prieto, 2017). Asimismo, Gusinde (1991a [1974], pp. 133-134) habla de pasos terrestres por los que circulaban kawésqar a pie, a través de los cuales se establecían nexos entre canoeros y cazadores terrestres (García, 2015).

En cuanto a conexiones de la zona archipelágica con el norte de la Isla Grande, hay referencias a pasos terrestres que implicaban un buen conocimiento cordillerano entre Harberton y el lago Fagnano (Bridges, 1951, p. 231; ver Pallo \& Borrazzo, 2016). Incluyen el paso que utilizó Anderson en 1902 de sur a norte (Skottsberg, 2004 [1911], p. 54) y Gusinde en el invierno de 1919 (Gusinde, 1982 [1937], p. 83), cuando debió retornar al Beagle por un problema en su pie.

\section{Caza de guanacos}

Para Orquera y Piana (1999b, p. 99) el guanaco es una "apropiada alternativa de los pinnípedos" e informan que hubo "momentos en los que los porcentajes de consumo llegaron a ser bastante altos", destacando sus ventajas, como su cuero, sus metapodios, su peso ("mayor 
que el de los Arctocephalus"), su médula o sus tendones, así como sus limitaciones, que incluyen la poca grasa -aunque esto parece un problema menor para quienes además comen lobos y ballenas ${ }^{4}$-, su baja densidad o la necesidad de rastrearlos (Orquera \& Piana, 2015, pp. 139140). Por su lado, Fernández y colaboradores (2018) marcan lo que llaman "different forms of interaction" con recursos terrestres a través del tiempo, básicamente el guanaco en la costa norte del canal Beagle. Para ello estudian los registros arqueofaunísticos de varios sitios $\mathrm{y}$, para tiempos posteriores a la Pequeña Edad del Hielo, concluyen que las condiciones más frías del período pueden asociarse con un decrecimiento en el consumo de guanacos (Fernández et al. 2018). En otras palabras, el período para el cual tenemos las observaciones etnográficas aquí mencionadas es en parte coincidente con un momento de baja explotación de guanaco, por lo que puede verse como una versión moderada de la importancia de ese recurso.

Con respecto a la obtención del guanaco Breivik y colaboradores (2016, p. 89) hablan de expediciones logísticas. Para el siglo XX Cristina Calderón menciona en qué forma el guanaco se iba a buscar "a los montes, cordillera arriba" (Zárraga, 2016, p. 37). Se ha destacado que no era una caza tan atractiva por lo penosa (Gusinde, 1986 [1937], p. 518; Orquera \& Piana, 2015, p. 140), situación que haría "verosímil que su captura haya sido considerada más 'costosa' que la de los pinnípedos [... pues] los guanacos debían ser esperados en silencio en medio del frío y la humedad del bosque, luego de heridos debían ser rastreados por terreno quebrado $y$ finalmente transportados a hombro hasta el campamento" (Orquera \& Piana, 1999a, p. 103). De todas maneras, es probable que el frío en la canoa no fuera menor, con el agregado de que ésta incluía la posibilidad de mojarse. Dentro de estas condiciones, la idea que ha imperado es la de caza invernal del guanaco en la costa (Orquera et al. 2012, p. 104). Esto también se relaciona con la distribución del guanaco, que tiende a bajar a la costa en invierno y remontar hacia zonas más altas o montañosas en verano.

4 Probablemente esto deba entenderse como un recurso que no se elegía como presa cuando existían alternativas con
Fitz Roy ha destacado que al este del Paso Murray (o sea, en Navarino) se cazaban guanacos en invierno y otras épocas del año; a su vez, el grupo de Orundelico, localizado al oeste del mismo no los cazaba (Fitz Roy, 2016 [1839], p. 174). Sin embargo, por la misma fuente se conoce que Orundelico cazó diez guanacos en la época de nieve en Wulaia, isla Navarino (Fitz Roy, 2016 [1839], p. 279). Entre otros casos se menciona que en agosto de 1879 algunos yaganes salieron a cazar guanacos en Ushuaia, con poco éxito (Chapman, 2010, p. 480). Las tasas de éxito informadas para los inviernos ca. 1870-1873 en dicho lugar, en que uno de cada cuatro o cinco cazadores conseguía un guanaco (Chapman, 2010, p. 460), no son tan malas, sobre todo considerando que ocurrían dentro de un sector muy disturbado por la Misión. Como destaca T. Bridges cazaron hasta unos $25 \mathrm{~km}$ de Ushuaia en julio de 1877 y capturaron cinco guanacos, lo que ha sido visto como una cosecha pobre (Orquera \& Piana, 2015, p. 140). Sin embargo, en términos comparativos, considerando el número de individuos implicados (14) y el número de días de caza (5), constituye un buen resultado para cazadores-recolectores (Speth, 2010, p. 88ss).

La valoración de la caza del guanaco no escapa al síndrome del cazador que, aunque no lo logra todos los días, con escasas presas puede ser más exitoso que quienes recolectan exitosamente (Lee, 1979; Hawkes et al. 1991). Es conocido que, por ejemplo, los cazadoresrecolectores San o los Shoshone tienen bajas tasas de éxito (Hitchcock et al. 1996; Thomas, 2015), particularmente de ungulados. Precisamente debido a que la caza es una actividad de alto riesgo y acompañada de poco éxito, se ha explicado su aparición sólo en el contexto de la existencia de otra fuente alimenticia garantizada, que son los recursos vegetales (Carmody, 2017, p. 315). Sin datos sistemáticos es difícil pronunciarse, pero las evidencias etnográficas en general no parecen escapar a la relación inversa entre éxito de caza y tamaño corporal de las presas que es característica de cazadores-recolectores (Lupo \& Schmitt, 2016). Por todo esto no son llamativas las tasas de éxito de caza de guanacos, sino que son normales. En

más grasa (mamíferos marinos). 
otras palabras, la incorporación de guanacos en la dieta puede ser sistemática e importante sin necesidad de que fueran presas cuantitativamente abundantes y es muy difícil minimizar su relevancia, particularmente si contribuyen a completar el ciclo anual, sin descuidar su posible significado en términos de la show-off hypothesis acerca de la ganancia social implicada (Hawkes, 1991). Por otra parte, sus eventuales dificultades de adquisición sólo aumentan las posibilidades de hambrunas.

A comienzos del siglo XX habitualmente se cazaban guanacos en invierno en Navarino (Orquera \& Piana, 2015, p. 139), pero también hay información previa. Desde la Beagle, se observó el 13 de mayo de 1830, "a crow's nest" en la costa sur de Navarino donde varios Yahgan estaban sentados esperando "to spear a guanaco" (Chapman, 2010, p. 142). Se informó que usan arpones o "spears", más efectivos que arcos y flechas (Martial, 2007 [1891], p. 34; Chapman, 2010, p. 142). El uso de arcos y flechas es referido por varias fuentes (Gusinde, 1986 [1937], pp. 446ss.; Fitz Roy, 2016 [1839], p. 172) y en general se asocia más con la caza en tierra que en el mar, aunque es ineludible la referencia a un impacto interpretado como de flecha en una vértebra de lobo hallada en el sitio Ajej I, con una cronología de 1.400 años AP (Piana et al. 2008; Orquera et al. 2012, pp. 63-65) ${ }^{5}$. En Ajej I también se recuperaron otras puntas interpretadas como de flecha (Piana et al. 2008). Además, debe mencionarse el uso de arco en los canales occidentales (Gusinde, 1991a [1974], pp. 274275; Duplessis, 2003 [1699-1701]; Skottsberg, 2004 [1911], p. 98; De Agostini, 2010 [1941], p. 86), Weddell vio arcos y flechas en el canal Bárbara que conecta el Pacífico con el Estrecho y Byron vio arcos en los cabos Froward y Quod (Emperaire, 1963 [1955], pp. 189-190). Se destaca que en el filme de Castelnau aparecen arcos hacia el año 1925 (Legoupil \& Chevallay, 2017, p. 75). En general Emperaire enfatiza que se los utilizaba para cazar guanacos, "pues esta arma se les hacía inútil cuando pasaban a los archipiélagos del oeste" (1963 [1955], p. 190). El arco y las flechas también han sido mencionados para la caza del huemul (Hippocamelus bisulcus) (Gusinde, 1991a [1974],

5 Anticipamos el registro de lo que puede verse como el caso opuesto, registrado en Brunswick, de una vértebra de pp. 274-275; De Agostini, 2010 [1941] p. 86). Al respecto Ratto (2003) ha destacado la existencia de una mayor diversidad de sistemas técnicos entre los yagán en comparación con los selk'nam, indicando el probable papel de la canoa facilitando el transporte de esta variedad de armas. Esta diversidad también puede implicar mecanismos de minimización de riesgo ante eventuales falencias en la adquisición de presas.

Un tema importante es la disponibilidad de guanacos en los distintos sectores del canal Beagle. Se ha sugerido que tenían una distribución limitada en el sur de la Isla Grande y creciente hacia el oriente de Ushuaia, como han sostenido Martial (2007 [1891], p. 40) o Gusinde (1986 [1937], p. 517). Sin embargo, el registro arqueológico informa que en ciertos casos localizados al oeste de Ushuaia se procesaron guanacos como en El Salmón -un sitio, por cierto, localizado a unos $2 \mathrm{~km}$ de la actual línea de costa en bahía Lapataia-, Bahía Golondrina, Río Pipo 17 (Figuerero Torres \& Mengoni Goñalons, 1986; Kligmann, 1992, 1995). Fernández y colaboradores (2018) analizaron las proporciones de valores de NISP de guanacos y otros recursos y disponen de tres casos -entre 28 arqueofaunas- que muestran al guanaco como dominante, nuevamente Isla El Salmón, Heshkaia 34 y Heshkaia 35 (Moat). Por otra parte, las excavaciones de Junius Bird en sus Rockshelter 1 (canal Murray, isla Navarino) y Rockshelter 3, bahía Yendegaia (Tierra del Fuego), presentaron frecuentes registros de instrumentos sobre guanaco con $8 \%$ y $15 \%$, respectivamente. Mas también abundaban registros no determinables de artefactos sobre Artiodacyla con $13 \%$ y $10 \%$, para una zona lejana a la distribución de huemul u ovinos de tiempos históricos o etnográficos (Morello et al. 2018). Más recientemente San Román y colaboradores (2018) registraron huesos de guanacos en un alero en Yendegaia, además de realizar numerosas observaciones de guanacos en toda esa región en octubre de 2017, a lo que se agregan nuestras reiteradas observaciones de grupos de guanacos, pilas de excrementos y concentraciones de huesos en febrero de 2020 (Fig. 4), o sea en verano, y las observaciones informadas al oeste de la Capitanía de Mar Punta Yámana (Cabo $2^{\circ}$ Jean Pizarro Tapia, com. pers., 2020).

huemul con una punta de arpón clavada (San Román et al. 2018). 


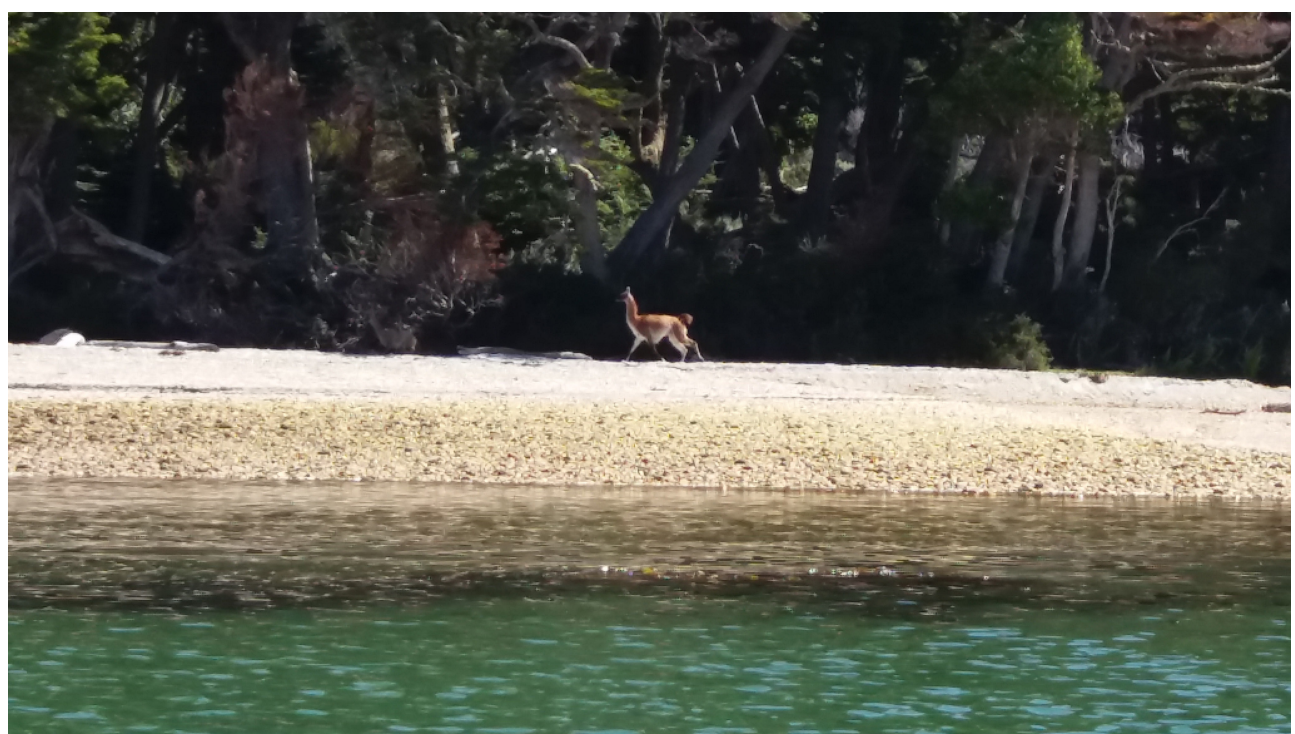

Fig. 4. Guanaco en la costa oeste de bahía Yendegaia (febrero de 2020). Fotografía de Luis Borrero.

Cristina Calderón, yagán, recuerda la abundancia de guanacos en Yendegaia hacia los años 1930 (Zárraga, 2016, p. 47). Pareciera que existen suficientes registros, actuales y pasados, que indican que la verdadera declinación de las poblaciones de guanacos ocurre bastante más al oeste de Ushuaia.

En cuanto a su importancia, debe destacarse que Orquera y Piana (1999b) presentan resultados de sondeos en 66 sitios; 35 de los cuales contienen restos de pinnípedos, 27 de guanacos y 15 de cetáceos. Esto sugiere una cierta relevancia de los guanacos; más aún, en 18 sitios aparecen tanto pinnípedos como guanacos. Más allá de esto, también al este hay hallazgos importantes de restos de guanacos (Yesner, 1990, 2004; Alunni \& Zangrando, 2012; Fernández et al. 2018). El 15\% de la fauna de Playa Larga son guanacos (Yesner, 1990, p. 14). En la localidad Heshkaia, ocupada en algún momento durante los últimos 1.600 años, el guanaco es dominante, incluyendo depósitos en que el número de individuos representados es sólo de uno o dos. En todos los casos se infiere transporte de partes (Alunni \& Zangrando, 2012; Alunni, 2018). En sintonía con esto, para el sitio Túnel VII, probablemente ocupado en el siglo XIX, Estévez Escalera y colaboradores hablan del "esporádico aprovechamiento del guanaco, para el cual es probable que la existencia de un área de 'reserva' en las faldas de la cordillera entre el área de la costa recorrida por los yámana y el área explotada regularmente por los cazadores pedestres del norte, fuera suficiente para reponer pérdidas" (1995, p. 182). Importa destacar la evidencia de alto consumo de guanaco brindada por el sitio Shamakush I (Orquera \& Piana, 1996). Aún más al este, en bahía Valentín, en la península Mitre, sectores probablemente menos relacionados con canoeros, los porcentajes de restos de guanacos recuperados en excavaciones son altos (Vázquez et al. 2011).

Para la zona occidental intermedia de los mares de Skyring y Otway, Joseph Emperaire destacó que los canoeros cazaban guanacos y que el huemul aparecía como alternativa (1963 [1955], p. 39). Todo esto parece robustecer la importancia del guanaco, principalmente en tiempos recientes. Algunos aspectos de la conducta de los canoeros iluminan la importancia del guanaco, tales como la costumbre de bañarse antes de comer el primer guanaco de primavera, información que escapa al modelo de caza invernal (Cojazzi, 1914 [1911], p. 108). Asimismo, en el caso de una inhumación canoera, en la isla Tres Mogotes, fechada en unos 500 años, uno de los cueros utilizados es de guanaco, en tanto que los cueros internos son de mamíferos marinos (Prieto \& Cárdenas, 2001).

Todo esto, incluyendo su importante rol estacional, nos lleva a postular que los guanacos constituían un recurso crítico en la subsistencia a lo largo del canal Beagle. 


\section{Caza de huemul}

Se trata más de un caso potencial que realizado. Lo cierto es que algunas de las razones para entrar al interior no se verifican en los archipiélagos del oeste. En especial las relacionadas con presas terrestres, que prácticamente no existían en las islas al oeste de Brecknock. Si bien hacia el norte del estrecho de Magallanes y al oeste de Fuego-Patagonia, el huemul era el vertebrado terrestre que podía ejercer atracción en el interior del continente, siempre se presentaba en densidades más bajas que el guanaco y sólo en ciertos sectores. Se reducían las posibilidades ofrecidas por las costas del continente en el Estrecho o los mares interiores $e$ islas del norte. Efectivamente, más al norte, comenta Achacaz Walakial, que los perseguían sólo cuando "necesitábamos buscar carne para comer" [...] “¡Ese es rico!” (Vega Delgado, 1995, pp. 31, 38) y, en términos más generales, los kawésqar cazaban lejos, donde "estaba el hábitat del ciervo" (Aguilera \& Tonko, 2013, p. 269). El sitio Jekchal, en Wellington, está caracterizado por ocupaciones tardías en las que el huemul es dominante como recurso.

Existe registro de huesos de huemul, aunque básicamente con una distribución costera y la mencionada punta ósea clavada en una vértebra lumbar de huemul en la costa sur de la península Brunswick (San Román, 2016; San Román et al. 2018). Además, se reitera su caza asociada a la estación invernal cuando bajaban a lugares cercanos a la costa, aunque actualmente también se divisan en las cercanías del litoral en toda época del año.

\section{Recorridos singulares}

Algunos recorridos tierra adentro han sido consignados en la literatura, sin que impliquen situaciones regulares. Por ejemplo, la ascensión del cabo Gloucester, en la más grande de las islas del grupo Grafton -Carlos- frente a la isla Santa Inés, fue realizada por canoeros en la mitad del tiempo que le tomó a Fitz Roy (Chapman, 2010, p. 114). También es pertinente la referencia de

6 Este último caso y varios otros localizados en grietas muy cercanas a la costa también trasuntan un grado de complejidad en el tratamiento de los muertos (Aspillaga \&
Achacaz Walakial (Vega Delgado, 1995, p. 65), quien cazando en tierra kawésqar encontró otros kawésqar "que estaban adentro de una cueva, en un pedrero", en una isla "mar afuera".

\section{Búsqueda de pigmentos}

Al no conocerse bien las fuentes de pigmentos sólo se puede especular que podían motivar desplazamientos hacia el interior. Ya se mencionaron fuentes potenciales en la costa (Fiore et al. 2008). Achacaz Walakial refiere a pigmentos que se obtenían "en las quebradas" (Vega Delgado, 1995 , p. 52) o el uso de arcillas "de las mesetas" (ib. p. 53). Para Orundelico se utilizaba una "sustancia ... recogida en el fondo de riachuelos de las montañas" para los pigmentos blancos (Darwin, 1906 [1839], p. 211). Martín González Calderón, yagán, destacó que el barro blanco usado en pinturas rupestres de la isla Sapinj es el mismo utilizado para pinturas corporales (2014, p. 178).

\section{Disposición de los muertos}

Fitz Roy (2016 [1839], p. 170) escribe acerca del traslado de cadáveres al bosque. La evidencia arqueológica de traslado de cadáveres a localizaciones alejadas de la costa incluye un entierro múltiple en el sitio Shamakush. Entierro a ca. $670 \mathrm{~m}$ de la costa, acompañado de evidencias que trasuntan complejidad social (Álvarez et al. 2008), el hallazgo de un párvulo a ca. 400-450 $m$ de la costa en Ajej I (Piana et al. 2006, 2008), dos individuos asociados con una fecha de $625 \pm 25$ años AP en un alero a $290 \mathrm{~m}$ en Mischihuen III (Piana et al. 2006) y cuatro individuos en el Sitio 130, un conchero en la ensenada Villarino, costa norte de la isla Navarino a unos 200 metros de la costa (Piana et al. 2006).

Más al norte de la región que nos ocupa se conoce el caso de un entierro en cuevas en el bosque en Última Esperanza, con una fecha de 250 \pm 65 años AP (Legoupil \& Prieto, 1991; Legoupil et al. 2004; Palacios \& Sierpe, 2019) 6

En general, las razones y posibilidades físicas para ir al interior crecen hacia el este,

Ocampo, 1996; Ocampo et al. 2000; Prieto \& Cárdenas, 2001; San Román \& Morello, 2001). 
según la disminución de la altura de las montañas. Entonces, un patrón que parece observarse es que el grado de variación en la interacción interiorcosta es mayor hacia el oriente, lo que llega a su culminación en la península Mitre, caracterizada por ocupaciones básicamente terrestres. Sin embargo, las ocupaciones registradas en la isla de los Estados, claramente marcando la existencia de medios de navegación (Horwitz, 1993), requieren mantener abiertas otras posibilidades.

\section{EQUIPAMIENTO}

\section{Equipamiento de individuos y de canoas}

El equipamiento de individuos entre canoeros puede mínimamente adoptar dos formatos, el material transportado por los individuos y el transportado en la canoa que, según las variantes de cada caso, regularmente implica mayor capacidad de transporte (Drennan, 1984). Chapman dice que los yámana "kept their material belongings to a minimum" (2010, p. 366). Los kawésqar transportan grasa de lobo en un trozo de tripa, que conservan 10-14 días (Gusinde, 1991a [1974], p. 330), o guardada "en un saquito de plumas de pájaros con las plumas hacia adentro" (Vega Delgado, 1995, p. 22). James Weddell registró, unos 20 años antes de la expedición de la Beagle, que algunos nativos que subieron a su barco, cerca de Cabo de Hornos, se atracaron "de vieja y rancia grasa de foca que llevaba varios días tirada" (2006 [1825], p. 154). La pirita es un clásico ejemplo de equipamiento de individuos, en este caso no siempre relacionado con abastecimiento directo. De acuerdo con Fitz Roy las mujeres llevan siempre pirita en una canasta y debido a que observa que no quieren desprenderse de ella, la juzga costosa (Fitz Roy, 2016 [1839], pp. 174-175). Lothrop publicó una fotografía de una bolsa yagán y su contenido, pedernal, yesca y pirita (1928, p. 129). A su vez, la bolsa de un cazador de la península Mitre contenía, a fines del siglo XIX, un raspador de vidrio enmangado en madera de "Fagus antarctica", un perforador de metal enmangado en un radio de ave, dos cuchillos de "acero" enmangados en madera de Berberis, tres formones de metal, dos

Esto sería así aunque la organización no se basara sobre almacenamiento para uso o consumo ya planificado y de ellos enmangados en Berberis y un afilador de arenisca (Outes, 1906).

Por supuesto, la canoa ofrece otras posibilidades de transporte. Skottsberg (1913, pp. 600) menciona que los kawésqar transportan en la canoa pedazos grandes de grasa, "heaps of mussels" -aunque cabe preguntarse si era para consumo o para depositarlos en la costa-, baldes de corteza para agua (Skottsberg, 1913, p. 600; Cooper, 1917, p. 205; Piana \& Orquera, 1998, p. 414), y Gusinde (1991a [1974], p. 330) señala que también llevan siempre algunas aves muertas y moluscos para tener comida. Además, la canoa yagán llevaba un fogón en el que mantenían fuego (Martial, 2005 [1888], pp. 232, 234). Ya mencionamos que Ratto (2003) destacó la importancia de la canoa para la diversidad de sistemas técnicos reconocidos entre los yagán.

\section{Equipamiento de lugares}

Las evidencias de equipamiento de lugares, en parte equivalentes a lo que Thomas (1985) llama depósito comunal, son las mismas que sustentan una geografía cultural organizada, planificada ${ }^{7}$. Casos de huesos de ballena o sus secciones funcionando como equipamiento o mobiliario de un lugar, por ejemplo, para usos como afiladores entre otros (ver Borrero, 2011, p. 291 para una observación de Hernán Vidal al respecto) y observaciones en Navarino. También interesa destacar el registro de la presencia de armas, tales como arpones enteros y/o sin daños aparentes, que sugieren potencial de reutilización que, según relato de Francisco Arroyo, kawésqar, se dejaban en el campamento para su uso a posteriori. Se trataba de armas que habían tenido mala suerte en procurar presas y debían ser abandonadas un tiempo para que perdieran dicha "mala suerte" (Francisco Arroyo, com. pers., 2009) [Fig. 5].

Chozas pre-preparadas. Es pertinente ante todo mencionar la evidencia arqueológica de casaspozo apud Bird de Punta Silvestre, isla Isabel, con remoción de arena de base (Borrero et al. 2019) o de Punta Santa Ana 1, con deschampado inicial,

se tratara de lo que Binford (1979) denominó Insurance Caching. 

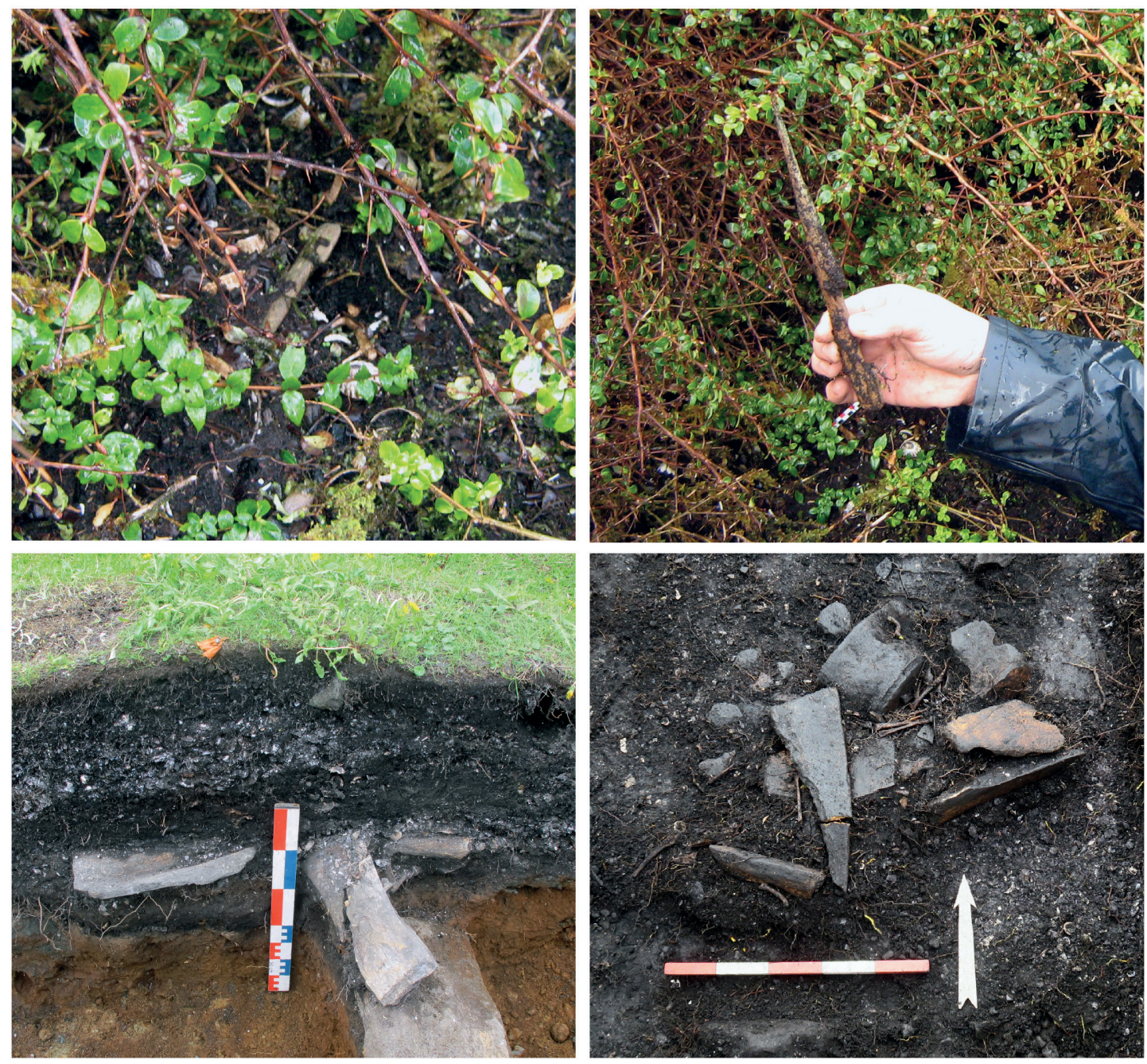

Fig. 5. Fila superior: arpón monodentado de espaldón simple hallado en superficie en caleta Fury.

Fila inferior: concentración de huesos de cetáceo en sitio Río Lum 36 (izquierda) y en Ukika 1 (derecha), costa norte de isla Navarino. Fotografías de Manuel San Román.

reconocido a partir de estudios micromorfológicos (Arroyo-Kalin \& French, 2012; Morello et al. 2012). La evidencia etnográfica es muy abundante. Bird computa ca. 50 armazones abandonados entre el golfo de Penas y el estrecho de Magallanes (Bird, 1946, p. 64; Gusinde, 1991a [1974], p. 174) y Gusinde menciona que los canoeros buscaban esos armazones para acortar los tiempos de instalación (Gusinde, 1991a [1974], p. 179; 1991b [1974], Figs. 3-5). De Agostini informa sobre armazones

8 Otros contextos con materiales de naufragios han sido ubicados, por ejemplo, en la bahía Valentín (Vázquez et al. 2011, p. 221), a lo que sin duda se agregarán los resultados en las islas Herschel y Hornos (1956, p. 203). Solari (1992) analizó los maderos de una choza en la isla Herschel y encontró que se utilizó madera de Pseudotsuga sp., procedente de un pecio norteamericano. Igualmente, se ha mencionado que una de las tempranas casas registradas en Mejillones fue en parte construida con maderos del Cervantes, hundido en 1930 (Zárraga, 2016, p. 98$)^{8}$. El estudio de las maderas de otras tres chozas analizadas por Solari, de las islas Wollaston

de la exploración sistemática de naufragios en la costa fueguina (Elkin et al. 2017). 
y Grévy, mostró el uso de Nothofagus betuloides, $N$. antarctica y Drimys winteri, maderas locales en todos los casos. Los armazones de chozas constituían un elemento conspicuo del paisaje archipelágico (Skottsberg, 2004 [1911], p. 101; Breivik et al. 2016, p. 87), pero existen otros elementos arqueológicos probablemente asociados con chozas que sugieren la existencia de otros atractores. Yesner observó en Playa Larga lo que denominó "ceremonial interments of both sea lion and particularly guanaco skulls, surrounded by a series of rocks" (1990, p. 18). En cabo Holland en el extremo occidental de bahía Wood, en el estrecho de Magallanes, se halló evidencia de un pozo que contenía una pila de vértebras de huemul, con las apófisis dorsales entrecruzadas, sobre el cual se formó un conchal (Johnson, 1976, p. 90). En un contexto completamente diferente fue registrada una situación similar en el continente, en el lago Argentino, con una concentración de cráneos de guanaco (Borrero, 2017). Lo que estos casos implican, más allá de sus posibles connotaciones trascendentales, es el acondicionamiento de lugares del espacio.

Corrales de pesca. La presencia de corrales de pesca en diversos puntos del archipiélago fueguino es bien conocida, pero existen pocas publicaciones que aborden su registro arqueológico, etnográfico y memoria oral. Tienen una extensa distribución espacial (ca. $41^{\circ}-56^{\circ} \mathrm{S}$ ) y se confeccionan a partir de un cerco o muro de piedras, ramas $y / o$ varas de madera, generalmente en zonas litorales de baja energía, desembocadura de ríos, humedales costeros y/o pozas del intermareal (Torres, 2009, 2013; Vázquez \& Zangrando, 2017). Requieren de un conocimiento específico de la relación entre emplazamientos costeros y las dinámicas locales de mareas. Además, el origen cultural y temporalidad (pre o post contacto) de esta tecnología de pesca es muy difícil de establecer, pero todos los registros apuntan a momentos muy tardíos del Holoceno. Se ha planteado que estas estructuras constituyen "una alta inversión de trabajo en la elaboración y mantenimiento del dique o corral, así como la participación de gran cantidad de personas" (Vázquez \& Zangrando, 2017, p. 103), lo que debe ponderarse con relación a su implicancia en la baja densidad de los grupos familiares de cazadores- recolectores. Estos autores mencionan variabilidad en la construcción que implica "piedras, ramas, estacas o una combinación entre éstas" (Vázquez \& Zangrando, 2017, p. 102). Las costas norte del canal Beagle presentan numerosos corrales similares a los descritos para Chiloé (R. Álvarez et al. 2008; Vázquez \& Zangrando, 2017) y son muy conocidas las estructuras de Wulaia, en la costa oeste de isla Navarino (Furlong, 1917, p. 180 y Figs. 5-6). También se las ha reconocido en islas al sudoeste de Brecknock (Torres \& San Román, 2018). Debe considerarse que en algunos casos los corrales pueden aparecer asociados con estructuras para el almacenamiento de moluscos (Torres \& San Román, 2018).

La edad de estas estructuras no es clara, pues muchas fuentes casi se restringen a repetir a Bridges (1951, p. 99). Por ejemplo, Hyades y Deniker dicen que Bridges les contó acerca de corrales de pesca, pero que ellos no los vieron (2007 [1891], p. 110). Gusinde se refiere sólo a nasas de palos y "manojos compactos de desbrozos" (1986 [1937], p. 534). La más clara referencia es la de Vázquez y Zangrando, quienes destacan que "la localización de estas estructuras en el intermareal sugiere que los casos aquí presentados corresponderían a momentos finales del Holoceno", destacando la "vigencia en la memoria oral" de corrales Yámana (2017, p. 137).

Refiriéndose a la parte occidental del archipiélago, Achacaz Walakial dice que para pescar acostumbraban "construir un corral antiguo" (Vega Delgado, 1995, p. 40) y hay otras referencias a por lo menos dos corrales en el canal David (Martinic, 2011, p. 57) y al uso de "empalizadas de ramas entrelazadas que construyen en las pequeñas angosturas de las bahías o en la desembocadura de ríos" (De Agostini, 2010 [1941], p. 86).

Destacamos también que los corrales de pesca, independiente de sus ambigüedades culturales y temporales, son una parte importante del equipamiento de lugares en la geografía fueguina y poseen una notable restricción archipelágica, pues están limitados a esta configuración de laberintos en islas, canales, fiordos, angosturas, etc. Como equipamiento de lugares, sus registros son marcadores de interacción cultural de tiempos tardíos a modernos. En este sentido, "las actividades ligadas a la pesca permiten ver una parte de la dinámica cultural del ámbito costero. 
Por ejemplo, el origen de los corrales de pesca de la costa noroeste de la isla [Tierra del Fuego] es un pequeño reflejo de la interacción cultural, en donde a lo largo de la prehistoria y posterior al contacto europeo se produjeron muchos $y$ variados encuentros interétnicos y de transmisión de tradiciones culturales, donde cada cual fue aportando en la construcción de este paisaje cultural litoral" (Torres, 2013, p. 238).

$\underline{\text { Porteos. }}$ Como se refirió más arriba, los porteos implican trabajo ingenieril e instalaciones asociadas con el cruce de islas, a veces hasta incluyendo almacenamiento de botes (Skottsberg, 1913), pero no siempre producen un registro material. Además, independiente de su materialidad, implican programación y un acabado conocimiento geográfico.

Varaderos. Se trata de depresiones $y$ montículos de rocas alargados, en sectores acotados y orientados perpendicularmente a la línea de costa, donde se ha despejado de rocas sobresalientes a fin de aplanar el sustrato intermareal donde se suben las canoas, arrastrándolas por la pendiente de la playa en forma de rampa (Fig. 6) y, a veces, se acondicionaban con algas. El proceso de limpieza genera un pseudo-muro tipo montículo por las acumulaciones alargadas de rocas de distinto tamaño entre las zonas despejadas. Hay abundantes descripciones de las rampas (Fitz Roy, 1839, frente a p. 326; Lothrop, 1928, p. 148 y Lám. XI; Gusinde, 1986 [1937], pp. 440, 596; Martial, 2007 [1891], p. 33). Furlong observó que los embarcaderos de canoas, limpios de rocas, se reconocían con marea baja (1917, p. 180 y Figs. 5-6). En la Lámina 29 publicada por Bajas Irizar (2007, p. 87), denominada "Fotografía de Martin Gusinde, 1919-1923. Archipiélago fueguino. Retrato de dos niños desnudos, en exterior, delante de un bote. Anthropos Institut. Sankt Augustin, Alemania", el bote parece estar descansando sobre un varadero. Para un sector ubicado más al occidente hay referencias a un varadero en la bahía Choiseul, isla Santa Inés (Martinic, 2011, p. 57) y otros casos como bahía del Águila en la zona central del estrecho de Magallanes y recientemente en la costa sur este de la isla Londonderry (obs. pers.).
Depósitos de almacenamiento. Las fuentes etnográficas registran técnicas de conservación de alimentos mayormente restringidas a casos de hongos y cetáceos, tanto para el oeste como para el canal Beagle. Sin embargo, también se menciona la conservación de carne de lobo (Gusinde, 1991a [1974], p. 330; Chapman, 2010, p. 94). Los hongos se conservan secos y tal vez se transportan a manera de comida de marcha (Lothrop, 1928, p. 30; Hyades \& Deniker, 2007 [1891], p. 88; Chapman, 2010, p. 94). Para la carne y grasa de ballena se registran depósitos en chorrillos de agua dulce, en charcos cubiertos con piedras, en turberas o enterrados en sedimentos intermareales (Orquera \& Piana, 2015, p. 195), mencionándose pozos de hasta $120 \mathrm{~cm}$ (Gusinde, 1991a [1974], p. 330). Estos depósitos son el resultado básicamente del carroñeo de animales varados (Chapman, 2010, pp. 178, 452, 465-466 para el canal Beagle y zona sur; Schindler, 1995 para península Mitre; Gusinde, 1991a [1974], pp. 185, 327-328 para el oeste), que muchas veces se consumían cuando se había iniciado la descomposición. Lo último adquiere pleno sentido dentro del marco expuesto por Speth (2017).

Tales depósitos parecen haber constituido anclas en el paisaje, para uso futuro. Desde ya implican la existencia de planeamiento y a menudo generaron grandes desplazamientos y concentraciones de individuos (Schindler, 1995). Estas observaciones han sido publicadas, casi siempre, dentro del contexto de la cooperación entre cazadores-recolectores, aunque Chapman (2010, p. 452) menciona un caso de ocultamiento de grasa para usarla en forma exclusiva. Este es un ejemplo local de una conducta observada reiteradamente en sociedades de cazadoresrecolectores (Boehm, 2017, p. 773).

Una interesante ironía es que son los recursos unearned los que llevan a casos más claros de planeamiento. El almacenamiento de recursos perecederos es, dada la falta de una preparación especial (sólo conocida para los hongos), una estrategia a corto plazo. El grado de conocimiento implicado en la elección de emplazamientos y uso de recursos almacenados que deberán ser utilizados por poblaciones dispersas es alto (Howey \& Frederick, 2016). Por otra parte, por más que el almacenamiento sea de baja inversión tecnológica, presenta costos de preparación. 


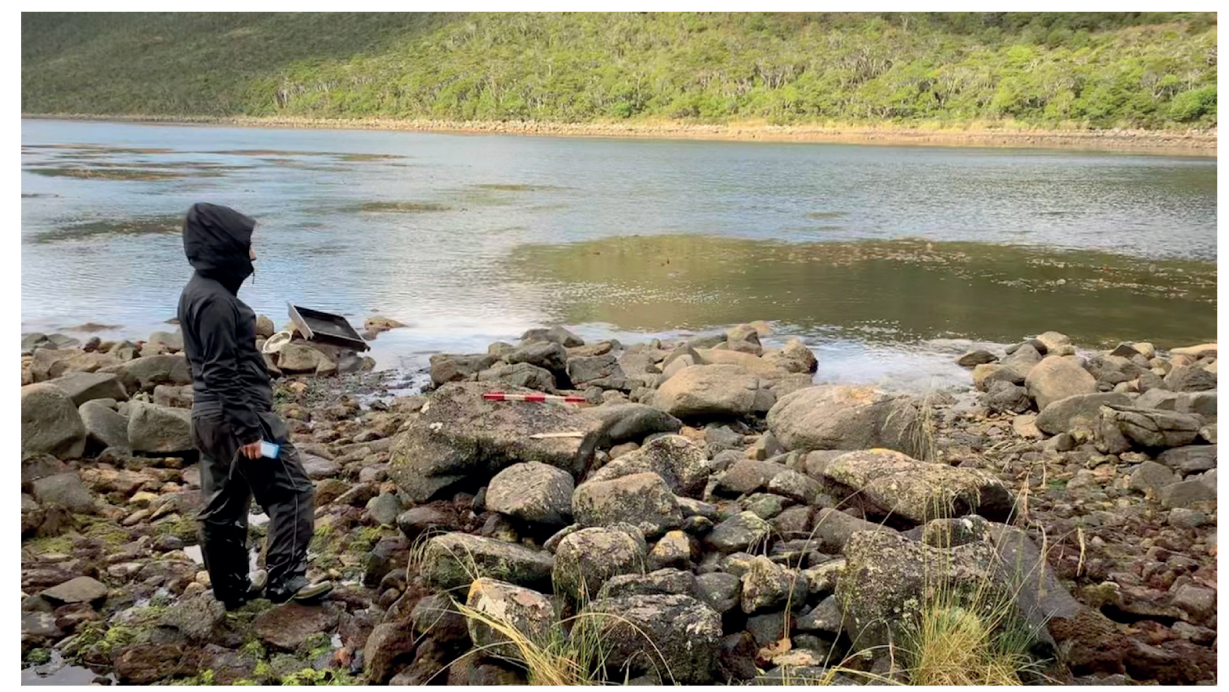

Fig. 6. Varaderos del archipiélago fueguino sudoccidental. Superior: dos varaderos en sitio arqueológico Londonderry

4 (2020); Centro: sitio

Bahía Mejillones 45 con gran montículo de conchal y dos varaderos (2016); Inferior: rasgo ubicado al sur de isla Desolación, canal Abra (2000). Fotografías de Flavia Morello.
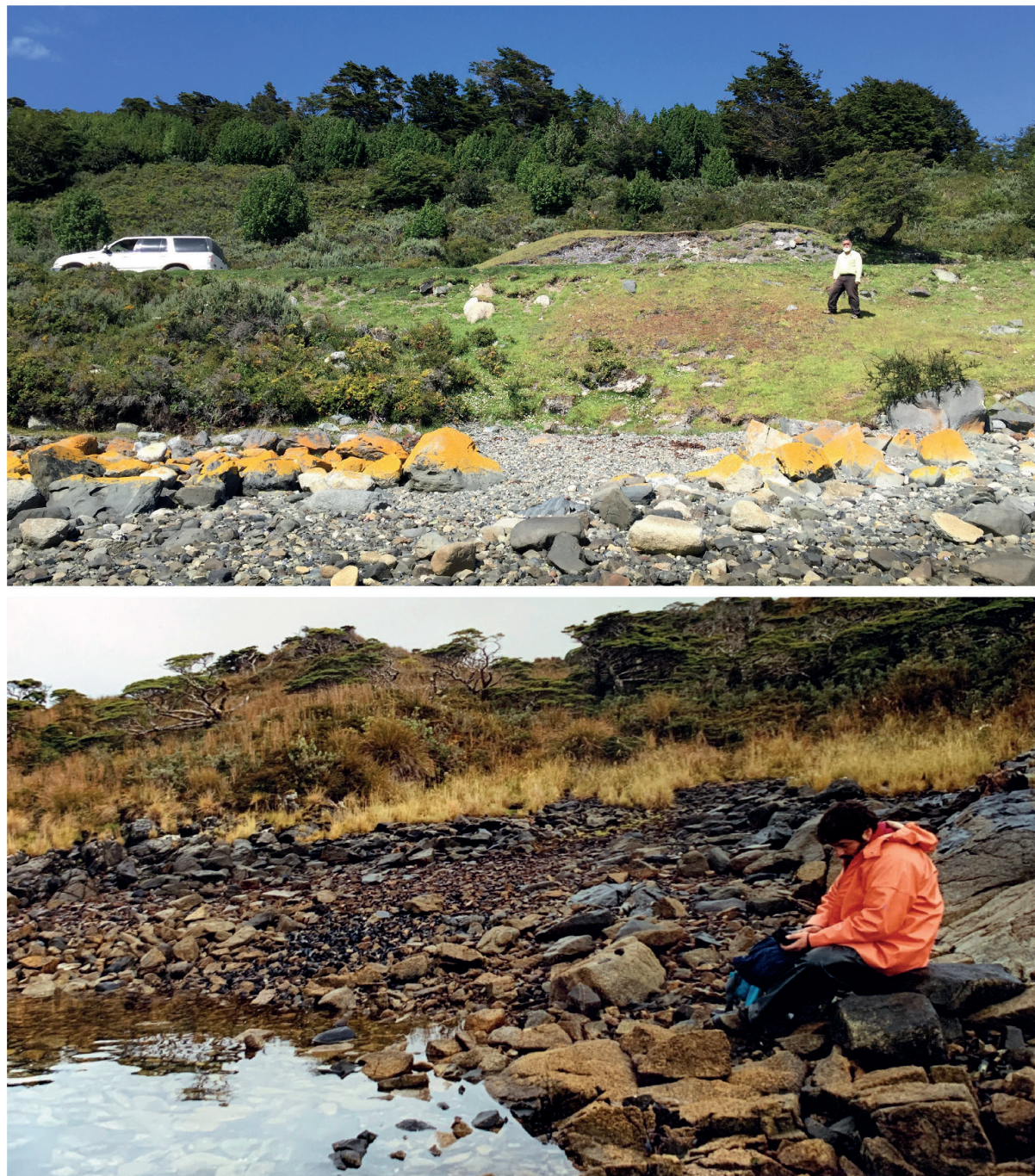
El tema arqueológico de los depósitos se asocia con una ulterior y conocida paradoja, ya que encontramos en general los depósitos que no fueron usados (Thomas, 1985). Pero indudablemente en al menos algunos casos hay otro significado y es que la seguridad del sistema fuera alta. Ningún depósito de alimentos ha sido recuperado en nuestra región, por lo que carecemos de ese control. Una consideración de interés es que la ubicación de los sitios tardíos (<2000 años AP) en la isla Navarino, que se sitúan en alturas importantes sobre el nivel del mar (Ocampo \& Rivas (2000) registran sobre 30 msnm y nosotros hemos visto sitios-conchales y restos domésticos por sobre 50 msnm aproximadamente), es adecuada como loci para conservación de carne y grasa. A esas alturas se registran descensos de temperatura promedio de $0,3^{\circ} \mathrm{C}$ (la temperatura baja $0,65^{\circ} \mathrm{C}$ cada 100 metros ascendidos). La búsqueda de altura como "efecto refrigerador" es un tema por investigar.

La evidencia arqueológica fueguina de depósitos se restringe a conjuntos de materiales líticos -lo que Hiscock (1989) llamaría "almacenamiento" ante la ausencia de evidencia de que estaban ocultos-, tales como el conjunto de 24 raspadores hallado en el sitio puesto Darwin en el canal Whiteside (F.M., obs. pers.) o los abundantes raspadores de cuarzo en el Cuarto Componente de Túnel 1, de ca. 2.700 años AP, estos últimos interpretados en función del procesamiento de pieles o cueros (Mansur \& Lasa, 2005, 2008; Orquera et al. 2012, p. 63). La lógica de esta clase de depósitos de materiales no perecederos es otra, se trata de equipamiento de lugares a largo plazo, conjuntos que pudieron ser utilizados y sin embargo permanecieron almacenados con posterioridad a su uso. El equipamiento de un lugar no implica necesariamente cantidades abundantes, pues hay bienes que si bien escasos, pueden no asociarse con transporte. Un ejemplo son los molinos o grandes núcleos preparados (especialmente rocas de buena calidad para la talla) que son dejados en un estado productivo y, en general, todos los bienes cuyo tamaño o peso hagan costoso su traslado. En este tema resulta de interés mencionar las evidencias de retomado de núcleos en sitios

La riqueza de alternativas de conducta, así como del technium correspondiente a canoeros (Orquera \& Piana, $1999 b, 2015)$ son condiciones que tornan implausibles las arqueológicos. Por ejemplo: Punta Santa Ana 3 y Cabo San Vicente, en donde se combina esta estrategia de almacenamiento in situ con otros aspectos de la planificación tecnológica como son diseños versátiles Levallois y estrategias expeditivas (Morello, 2005, 2016).

Algunos de los bienes que equipan el espacio pueden pasar a constituir equipamiento de individuos, sobre todo cuando se acerca el final de su vida útil, o sea, cuando se vuelven transportables. Ese puede ser el caso de la toba Miraflores o de fragmentos de arenisca utilizados como afiladores o alisadores.

\section{CONCLUSIONES}

Hemos visto que existe un cuadro de alta variabilidad arqueológica manifestada en el canal Beagle y zonas al este (Orquera \& Piana, 1999a; Tivoli \& Zangrando, 2011; Vázquez et al. 2011; Zangrando et al. 2016, 2017) y tan sólo elementos aislados acerca de la variación hacia el oeste y sur. La suma de toda esta información da una medida de la amplitud efectiva del nicho ecocultural para tiempos relativamente recientes, incluyendo instancias de reorientación en el uso del ambiente y argumentos sobre "offshore-going practices and the growth in ranges of maritime mobility" (Tivoli \& Zangrando, 2011, p. 1148), en este caso siempre a la vista de la costa. La falta de contemporaneidad entre las muchas observaciones y datos aquí utilizados aseguran que nuestro esquema encubre variación temporal. Entre muchas otras preguntas temporales, se destaca la necesidad de conocer la edad de los corrales de pesca y varaderos. Muchos patrones registrados en la literatura seguramente tienen un componente temporal, tales como los posibles tabúes alimenticios relacionados con peces (Fiore \& Zangrando, 2006) o con zifios (Orquera \& Piana, 2015, p. 189), al igual que procesos tales como el de intensificación (Zangrando, 2009). Asimismo, cabe preguntarse si el uso intensivo de ocre para pinturas corporales (Fiore, 2005), o para pinturas rupestres, implicó un cambio de medio dentro de lo que se denomina señalamiento costoso (Bird \& O'Connell, 2006) ${ }^{9}$. Igualmente

consideraciones sobre la potencial pérdida de técnicas de confección de vestimentas (Garvey, 2018). 
surge la necesidad de saber si las pequeñas diferencias observadas a través del tiempo en la importancia de distintos recursos reflejan cambios de estrategia o meramente de tácticas, en particular para el Holoceno tardío (San Román, 2016). En otras palabras, un mayor refinamiento cronológico será necesario para avanzar en las discusiones aquí planteadas.

Varios observadores históricos transmitieron la existencia de ciclos anuales para los canoeros, entre ellos Martial, quien destaca que en verano "se diseminan en las pequeñas islas desboscadas próximas a la costa para recoger los huevos que las aves ponen ... buscan al mismo tiempo en las selvas la corteza de haya ... para componer sus piraguas ... Durante el invierno, los yahganes se refugian en los innumerables canales de la costa, alimentándose ... principalmente de mariscos" (2005 [1888], p. 226). Sin duda, ciclos como los mencionados refieren a actividades características de la región, pero los mismos son excesivas simplificaciones. El trabajo de Legoupil (19931994) mostró el uso más especializado de sectores geográficamente marginales. Hacia el oeste se conoce mucho menos y se puede postular que otros ciclos pueden estar encriptados en la escasa información disponible.

En un nivel más general, Fitzpatrick y colaboradores (2016) se plantearon si las islas más pequeñas deben siempre ser consideradas como marginales dentro de un sistema. Se ha observado -principalmente con ejemplos del Pacífico central y el Caribe, incluyendo islas continentales, no exclusivamente oceánicas- que en muchos casos las islas más pequeñas concentran más recursos y resultan más atractivas para la instalación humana (Keegan et al. 2008; Fitzpatrick et al. 2016). En nuestra región, más allá de que el tamaño actual de las islas es, en muchos casos, mayor al que tuvieron durante la transgresión holocena, no parece ser el caso, por lo que los datos no apoyan la propuesta de Fitzpatrick y colaboradores. Es tan escasa la investigación en las islas pequeñas (Ortiz Troncoso, 1972; Horwitz, 1993; Legoupil \& Fontugne, 1997; San Román \& Morello, 2001; Muñoz et al. 2016) que debe aún quedar abierta la discusión de hasta qué punto algunas de ellas formaron parte

10 Lo que puede chocar con la inferencia de Garvey (2018) acerca de la poca conectividad de los Yámana en del programa de poblamiento original del área de Cabo de Hornos, quizá a manera de stepping stones (MacArthur \& Wilson, 1967, p. 29), siempre teniendo en mente que su tamaño es inferior al de fines del Pleistoceno (Ponce et al. 2011). Es muy claro que algunas de estas islas se ubican fuera de los circuitos de poblamiento temprano teóricamente esperables, como las Wollaston, pero todas las del borde del Pacífico -mínimamente exploradasabren una interrogante. Esto se destaca a la luz de las recientes posiciones acerca del poblamiento de América por la costa Pacífica (Dixon, 2013; Braje et al. 2020), en que islas relativamente pequeñas ya han probado su relevancia para el poblamiento (Fedje \& Christensen, 1999; Erlandson et al. 2011). Las islas Wollaston o Hermite eran de uso parcial, probablemente estacional (Ortiz Troncoso, 1972; Legoupil, 1993-1994). Su aislamiento creaba condiciones para que durante tormentas quedaran aislados grupos sociales, con peligro de morir de hambre. Vale recordar lo que escribió Barry Smith, "we realized how easy it would be to remain trapped among the Wollastons" (1979, p. 14). En otras palabras, esas islas eran efectivamente marginales en términos de intensidad ocupacional y de conectividad. Para otras islas, aun en casos en que se habla de aislamiento, las mismas estaban ubicadas dentro de un cuadro de conectividad, que permitía capear temporales. Ahora bien, lo que Martial llama "un aislamiento casi continuo" de la región central (2005 [1888], p. 226), no sólo probablemente era inferior al que podía ocurrir más al sud-oeste en los bordes del Pacífico, sino que funcionaba dentro del rango de muchos otros grupos o bandas. Esto quiere decir que la región central presentaba alta conectividad ${ }^{10}$. Todos estos casos, junto con el planteado por el cruce del canal Beagle, u otros canales menores, invitan a considerar el "commuter effect" (Keegan \& Diamond, 1987, p. 59), o sea, casos de islas muy pequeñas para sostener una población, pero que pueden ser habitables y que se usan si están a distancia de comunicación de otra isla o del continente.

Toda la información aquí rápidamente resumida acerca de alternativas de distribuciones de poblaciones de guanacos y otros recursos

comparación con los Unangan de las islas Aleutianas. 
-principalmente pinnípedos (Schiavini, 1993; San Román, 2016)-, unida al importante papel otorgado a los unearned resources, sopesado en relación con la cada vez más abundante información arqueofaunística, deberá permitir subdivisiones significativas dentro del ya muy variado bloque espacio-temporal de aproximadamente los últimos 1.000 años en la región aquí tratada (Santiago \& Vázquez, 2009, p. 235). De cualquier manera, un patrón que tiene posibilidades de permanecer es el que muestra que la alta movilidad no debe relacionarse necesariamente con falta de planificación. La ampliación del espectro de sitios y contenidos existentes en escala regional muestra una gran variedad. Ante todo, debemos imaginar, para al menos segmentos de ese período, un paisaje caracterizado por anclas, tales como depósitos de carne o grasa en turberas, intermareales $y$ chorrillos, corrales de pesca o pinturas rupestres, que pueden o no asociarse con la conectividad programada, indicada por porteos, varaderos de canoas o lugares de alimentación al paso y hasta quizá depósitos de instrumentos en anticipación de tareas. Estas son las expresiones materiales que deben asociarse con los tipos de sitios más conocidos, concheros, lugares de abastecimiento de rocas o campamentos de caza en el interior. Una característica destacada de muchos de estos elementos es que implican técnicas ingenieriles y por ello, aun en los casos de baja inversión tecnológica, equipan el espacio. Entonces, no es el acceso primario a recursos alimenticios el exclusivo imán de la movilidad humana canoera, sino que ésta responde a un espacio preparado para los desplazamientos, con una serie de lugares preestablecidos. Esto es lo mismo que decir que había patrones de movimiento programado. El funcionamiento de estos sistemas debió contemplar mecanismos para una serie de situaciones posibles ("riesgo") y seguramente disponer de respuestas para lo inesperado ("incertidumbre"). También puede defenderse la existencia de lo que Binford ha llamado "visiting zone" para los Nunamiut (1982), en el caso fueguino claramente funcionando en una escala supra-étnica. Esto significa que no necesariamente debieron utilizarse todas las tácticas y estrategias, o inclusive todas las posibilidades del technium al mismo tiempo. Todos estos ritmos admiten como mínimo variaciones según las condiciones previas, contemporáneas y posteriores a la Misión Anglicana de Ushuaia, o en sus localizaciones posteriores. Tanto tecnologías vigentes como latentes o respuestas circunstanciales innovativas pudieron conformar esa biblioteca de conocimientos utilizados por los canoeros para mantenerse en el largo plazo. Las condiciones de programación, particularmente las inferidas de las actividades ingenieriles, contribuyen a fundamentar el concepto de adaptación exitosa atribuido a los canoeros.

En fin, se abren más interrogantes, sistematizando el concepto de geografía cultural y jerarquizando el espacio de acuerdo con valores culturales que dispongan de visibilidad y obstrusividad arqueológica. Asimismo, este concepto permite ampliar las discusiones de las dinámicas de organización, instalación y circulación humana en los paisajes fueguinos del meridión.

\section{AGRADECIMIENTOS}

Este trabajo fue realizado bajo los auspicios de proyecto FONDECYT 1190984. Agradecemos a todos los colegas que respondieron a nuestras consultas o generosamente compartieron información muchas veces inédita: Juan Bautista Belardi, Karen Borrazzo, Miguel Chapanoff, Denis Chevallay, María José Figuerero Torres, Dánae Fiore, Débora Kligmann, Daniel R. Martinioni, Ernesto L. Piana, Victor Sierpe, John D. Speth, Jimena Torres, Martín Vázquez y Francisco Zangrando. Igualmente agradecemos la información brindada por el Cabo $2^{\circ}$ Jean Pizarro Tapia y las detalladas observaciones de los evaluadores que ampliaron el alcance de nuestro trabajo.

\section{BIBLIOGRAFÍA}

Aguilera, O. E., \& Tonko, J. (2013). Relatos de viaje kawésqar. Temuco: Ofqui editores.

Alunni, D. (2018). Estrategias de aprovisionamiento de guanacos y uso del bosque Magallánico por cazadoresrecolectores costeros. Revista del Museo de Antropología, 11(2), 7-22.

Alunni, D., \& Zangrando, A. (2012). Primeros datos sobre el transporte, procesamiento y consumo de guanacos en la localidad arqueológica Heshkaia (sudeste de Tierra 
del Fuego, Argentina). Magallania, 40(1), 319-331.

Álvarez, M., Vázquez, M., \& Piana, E. (2008). Prácticas mortuorias entre los cazadores-recolectores del canal Beagle: el caso de Shamakush entierro. Magallania, 36(2), 105-121.

Álvarez, M., Pal, N., Salvatelli, L., Briz i Godino, I., Zangrando, F. A., Bazerque, A., De Angelis, H., \& Fiore, D. (2010). Tallando desde lo alto: un sitio de explotación de materiales líticos de los grupos cazadores-recolectores de la Isla Grande de Tierra del Fuego. Magallania, 38(1), 295-300.

Álvarez, R., Munita, D., Fredes, J., \& Mera, R. (2008). Corrales de pesca en Chiloé. Consejo Nacional de la Cultura y las Artes, Valdivia.

Anónimo (1992). Catálogo del Museo Regional Salesiano Maggiorino Borgatello. Punta Arenas.

Arroyo-Kalin, M., \& French, C. (2012). Micromorphological analysis of archaeological shell middens and buried soils at Punta Santa Ana and Pizzulic-2, Magallanes, Chile. Informe Final, Proyecto FONDECYT 1085329. Ms.

Aspillaga, E., \& Ocampo, C. (1996). Restos óseos humanos de la Isla Karukinka (Seno Almirantazgo, Tierra del Fuego). Informe preliminar. Anales del Instituto de la Patagonia (Serie Ciencias Humanas), 24, 153-161.

Bajas Irizar, M. P. (2007). Estrategias y relatos visuales en los álbumes fotográficos de Tierra del Fuego. En M. Alvarado, C. Odone, F. Maturana \& D. Fiore (Eds.), Fueguinos. Fotografías Siglos XIX y XX. Imágenes e Imaginarios del Fin del Mundo (pp. 75-88). Santiago de Chile: Pehuen.

Binford, L. R. (1979). Organization and Formation Process: Looking at Curated Technologies. Journal of Anthropological Research, 35, 255-273.

Binford, L. R. (1982). The Archaeology of Place. Journal of Anthropological Archaeology, 1, 5-31.

Bird, J. (1932). Tierra del Fuego 1932-1933. Letters 1934-37. AMNH, Ms.

Bird, J. (1946). The Alacaluf. In J. H. Steward (Ed.), Handbook of South American Indians (Vol. 1, pp. 55-79). Bureau of American Ethnology Bulletin 143, Smithsonian Institution, Washington, D.C.

Bird, D. W., \& O'Connell, J. F. (2006). Behavioral ecology and archaeology. Journal of Archaeological Research, 14, 143-188.

Boehm, C. (2017). Ancestral Precursors, Social Control, and Social Selection in the Evolution of Morals. In M. N. Muller, R. W. Wrangham \& D. R. Pilbeam (Eds.), Chimpanzees and Human Evolution (pp. 746-790). Cambridge: The Belknap Press of Harvard University
Press.

Borrazzo, K. (2012). Raw material availability, flaking quality and hunter-gatherer decision making in Northern Tierra del Fuego Island (Argentina). Journal of Archaeological Science, 39, 2643-2654.

Borrazzo, K., Borrero, L. A., \& Pallo, C. (2019). Exploring lithic transport in Tierra del Fuego (Southern South America). Journal of Archaeological Science: Reports, 24, 220-230.

Borrero, L. A. (1985). Un modelo de ocupación humana de la región del Seno de la Última Esperanza (Magallanes, Chile). Publicaciones del Instituto de Antropología, 38-39, 155-171.

Borrero, L. A. (1991). Los 'modelos de situaciones excepcionales' y el estudio de las sociedades de cazadores y recolectores. Comechingonia, 8(7), 107127.

Borrero, L. A. (2011). Los cazadores orientales de Tierra del Fuego. En A. F. Zangrando, M. Vázquez \& A. Tessone (Eds.), Los cazadores-recolectores del extremo oriental fueguino Arqueología de Península Mitre e Isla de los Estados (pp. 287-298). Sociedad Argentina de Antropología, Buenos Aires.

Borrero, L. A. (2017). Forests, steppes, and coastlines: zooarchaeology and the prehistoric exploitation of Patagonian habitats. In U. Albarella, M. Rizzetto, H. Russ, K. Vickers \& S. Viner-Daniels (Eds.), The Oxford Handbook of Zooarchaeology (pp. 674-687). Oxford: Oxford University Press.

Borrero, L. A., \& Borrazzo, K. (2011). La geografía cultural del sudoeste de Patagonia continental. En L. A. Borrero \& K. Borrazzo (Eds.), Bosques, montañas y cazadores. Investigaciones Arqueológicas en Patagonia Meridional (pp. 7-36). CONICET-IMHICIHU, Buenos Aires.

Borrero, L. A., \& Borrazzo, K. (2016). Exaptaciones, cambio y oportunismo en arqueología. Revista de Cazadoresrecolectores, 7, 9-29.

Borrero, L. A., Morello, F., San Román, M., McEwan, C., \& Martin, F. M. (2019). Isla Isabel: Viajeros, registros arqueológicos y geografía cultural. Magallania, 47(1), 117-144.

Braje, T. J., Erlandson, J. M., Rick, T. C., Davis, L., Dillehay, T., Fedje, D. W., Froese, D., Gusick, A., Mackie, Q., McLaren, D., Pitblado, B., Raff, J., Reeder-Myers, L., \& Waters, M. R. (2020). Fladmark + 40: What Have We Learned about a Potential Pacific Coast Peopling of the Americas? American Antiquity, 85(1), 1-21.

Breivik, H. M., Bjerck, H. B., Zangrando, A. F. J., \& Piana, 
E. L. (2016). On the applicability of environmental and ethnographic reference frames: an example from the high-latitude seascapes of Norway and Tierra del Fuego. In H. B. Bjerck, H. M. Breivik, S. E. Fretheim, E. L. Piana, B. Skar, A. M. Tivoli \& A. F. J. Zangrando (Eds.), Marine Ventures (pp. 75-94). Equinox, Sheffield.

Bridges, E. L. (1951). The Uttermost Part of the Earth. London: Hodder and Stoughton.

Bridges, E. L. (1952). El último confín de la tierra. Buenos Aires: El Ateneo.

Bruchhausen, P. (1966). Isla Santa Inés, Patagonia. American Alpine Journal, 15(1), 185.

Byron, J. (1996 [1768]). Naufragio en las costas patagónicas. Buenos Aires: Ediciones del Sol.

Campbell, A. (2012 [1747]). Continuación del viaje a los mares del sur. En Cuatro Relatos para un Naufragio. La fragata Wager en el golfo de Penas en 1741 (pp. 231-281). Santiago de Chile: Septiembre Ediciones.

Carmody, R.N . (2017). Evolution of the Human Dietary Niche. Quest for High Quality. In M. N. Muller, R. W. Wrangham \& D. R. Pilbeam (Eds.), Chimpanzees and Human Evolution (pp. 311-338). Cambridge: The Belknap Press of Harvard University Press.

Chapman, A. (2010). European Encounters with the Yamana People of Cape Horn, Before and After Darwin. Cambridge: Cambridge University Press.

Chevallay, D. (1999). Una ruta terrestre entre el seno Almirantazgo y el canal Beagle: indicios de intercambios entre las etnias fueguinas. Ms.

Christensen, M. (2016). La industria ósea de los cazadoresrecolectores: el caso de los nómades marinos de Patagonia y Tierra del Fuego Chile. Punta Arenas: Ediciones Universidad de Magallanes.

Cojazzi, A. (1914 [1911]). Gli indii dell' arcipelago fueghino: Contributi al folk-lore e all' etnografia dovuti alle missioni salesiane. /en español/ Revista Chilena de Historia y Geografía, IX, 288-352; X, 5-51.

Coles, J., Hibbert, F., Orme, B., Pettit, M., Rushton, D., \& Switsur, V. (1973). Prehistoric Roads and Tracks in Somerset, England: 3. The Sweet Track. Proceedings of the Prehistoric Society, 39, 256-293.

Cooper, J. M. (1917). Analytical and critical bibliography of the tribes of Tierra del Fuego and adjacent territory. Bureau of American Ethnology. Bulletin 63. Smithsonian Institution, Washington D.C.

Darwin, C. (1906 [1839]). Journal of Researches into the Geology \& Natural History of the Various Countries Visited During the Voyage of the Beagle Round the World. London: Dent \& Sons.
De Agostini, A. (1956). Treinta años en Tierra del Fuego. Buenos Aires: Peuser.

De Agostini, A. M. (2010 [1941]). Andes Patagónicos. Viajes de exploración a la cordillera patagónica austral. Congregación Salesiana de Chile, Punta Arenas.

Dixon, E. J. (2013). Late Pleistocene colonization of North America from Northeast Asia: new insights from largescale paleogeographic reconstructions. Quaternary International, 285, 57-67.

Drennan, R. (1984). Long-Distance Transport Costs in PreHispanic Mesoamérica. American Anthropologist, 86(1), 105-112.

Duplessis, R. S. (2003 [1699-1701]). Périple de Beauchesne à la Terre de Feu (1698-1701), une expédition mandatée par Louis XIV. Paris: Éd. Transboréal.

Elkin, D., Murray, C., \& Grosso, M. (2017). Arqueología de naufragios históricos en la costa atlántica fueguina. En M. Vázquez, D. Elkin \& J. Oría (Eds.), Patrimonio a orillas del mar. Arqueología del Litoral Atlántico de Tierra del Fuego (pp. 103-145). Ushuaia: Editora Cultural Tierra del Fuego.

Emperaire, J. (1963 [1955]). Los nómades del Mar. Santiago: Ediciones de la Universidad de Chile.

Erlandson, J. M., Rick, T.C., Braje, T. J., Casperson, M., Culleton, B., Fulfrost, B., García, T., Guthrie DA, Jew N, Kennett DJ, Moss ML, Reeder L, Skinner C, Watts J, Willis L. (2011). Paleoindian seafaring, maritime technologies, and coastal foraging on California's Channel Islands. Science, 441, 1181-1185.

Estévez Escalera, J., Juan-Muns Plans, N., Martínez Moreno, J., Piqué Huerta, R., \& Schiavini, A. (1995). Zooarqueología y antracología: estrategias de aprovechamiento de los recursos animales y vegetales en Túnel VII. En J. Estévez Escalera \& A. Vila Mitjá (Eds.), Encuentros en los conchales fueguinos (pp. 143-193). Barcelona: Treballs d'Etnoarqueologia I, CSIC.

Fedje, D. W., \& Christensen, T. (1999). Modeling paleoshorelines and locating early Holocene coastal sites in Haida Gwaii. American Antiquity, 64, 635-652.

Fernández, M., Ponce, J. F., Zangrando, F. J., Borromei, A. M., Musotto, L. L., Alunni, D., \& Vázquez, M. (2018). Relationships between terrestrial animal exploitation, marine hunter-gatherers and palaeoenvironmental conditions during the Middle-Late Holocene in the Beagle Channel region (Tierra del Fuego). Quaternary International, doi.org/10.1016/j. quaint.2018.05.032

Figuerero Torres, M. J., \& Mengoni Goñalons, G. (1986). 
Excavaciones arqueológicas en isla El Salmón (Parque Nacional de Tierra del Fuego). PREP Informes de Investigación, 4, 1-94.

Fiore, D. (2005). Pinturas corporales en el fin del mundo. Una introducción al arte visual Selk'nam y Yamana. Chungara, Revista de Antropología Chilena, 37(2), 109-127.

Fiore, D., \& Zangrando, A. F. J. (2006). Painted Fish, eaten Fish: Artistic and archaeofaunal representations in Tierra del Fuego, Southern South America. Journal of Anthropological Archaeology, 25(3), 371-389.

Fiore, D., Maier, M., Parera, S.D., Orquera, L., \& Piana, E. (2008). Chemical analyses of the earliest pigment residues from the uttermost part of the planet (Beagle Channel region, Tierra del Fuego, Southern South America). Journal of Archaeological Science, 35, 3047-3056.

Fitzpatrick, S. M., Thompson, V. D., Poteate, A.S., Napolitano, M.F., \& Erlandson, J.M. (2016). Marginalization of the Margins: The Importance of Smaller Islands in Human Prehistory. The Journal of Island and Coastal Archaeology, 11(2), 155-170.

Fitz Roy, R. (1839). Narrative of the surveying voyages of His Majesty's Ships Adventure and Beagle between the years 1826 and 1836, describing their examination of the southern shores of South America, and the Beagle's circumnavigation of the globe. Proceedings of the second expedition, 1831-36, under the command of Captain Robert Fitz-Roy, R.N. London: Henry Colburn.

Fitz Roy, R. (2016 [1839]). Los viajes del Beagle. Informes de la segunda expedición (1831-1836). Buenos Aires: EUDEBA.

Furlong, C. W. (1917). Tribal distribution and settlements of Fueguians, comprising nomenclature, etymology, philology, and population. The Geographical Review, III(3), 169-187.

Gallardo, F., Ballester, B., Prieto, A., Sepúlveda, M., Gibbons, J., Gutiérrez, S., \& Cárcamo, J. (2018). Fuegian Firestone Quarry Iron Pyrite on Capitán Aracena Island, Magallanes Archipelago, Southern Chile. Current Anthropology, 59(4), 455-461.

García, S. (2012). Reinaldo Catalán y Francisco Oyarzún, baqueanos del sur de Tierra del Fuego (Chile) y su participación en la senda de penetración VicuñaYendegaia. Magallania, 40(1), 63-91.

García, S. (2015). Los orígenes de las comunicaciones terrestres en el sur de Tierra del Fuego (Chile). Magallania, 43(2), 5-43.
García, C., \& Prieto, A. (2017). Pasos de indios y límites étnicos tardíos en la Cordillera Darwin (Tierra del Fuego, Chile). Décimas Jornadas de Arqueología de la Patagonia, Libro de Resúmenes (p. 15). Ideas-CONICET, Puerto Madryn.

Garvey, R. (2018). Cultural Transmission and Sources of Diversity. A Comparison of Temperate Maritime Foragers of the Northern and Southern Hemispheres. In A. K. Lemke (Ed.), Foraging in the Past: Archaeological Studies of Hunter-Gatherer Diversity (pp. 19-48). Louisville: University Press of Colorado.

Gibbons, J., \& Harambour, S. (2016). Esclarecen el misterio de la piedra del fuego de los indígenas de nuestra Patagonia. La Prensa Austral, viernes 1 de enero de 2016.

González Calderón, M., Gañán, M., \& Serrano, A. (2014). Primer registro de arte rupestre en Tierra del Fuego. Magallania, 42(2), 175-181.

Gusinde, M. (1982 [1937]). Los indios de Tierra del Fuego. Tomo I (I) Los Selknam. Buenos Aires: CAEACONICET.

Gusinde, M. (1986 [1937]). Los indios de Tierra del Fuego. Tomo II (I). Los Yámana. Buenos Aires: CAEACONICET.

Gusinde, M. (1991a [1974]). Los indios de Tierra del Fuego. Tomo III (I). Los Halakwulup. Buenos Aires: CAEACONICET.

Gusinde, M. (1991b [1974]). Los indios de Tierra del Fuego. Tomo III (II). Los Halakwulup. Buenos Aires: CAEACONICET.

Hawkes, K. (1991). Showing off: Tests of an Hypothesis about Men's Foraging Goals. Ethology and Sociobiology, 12(1), 29-54.

Hawkes, K., O’Connell, J. F., \& Jones, N. B. (1991). Hunting income patterns among the Hadza: big game, common goods, foraging goals and the evolution of the human diet. Philosophical Transactions: Biological Sciences, 334 (1270), 243-251.

Hiscock, P. (1989). The Concept of Cache: A Reply to Moorwood. Archaeology in Oceania, 24(1), 38-39.

Hitchcock, R. K., Yellen, J. E., Gelburd, D. J., Osborn, A. J., \& Crowell, A. L. (1996). Subsistence hunting and resource management among the Ju/hoansi of northwestern Botswana. African Study Monographs, 17(4), 153-220.

Horwitz, V. D. (1993). Maritime settlement patterns: Isla de los Estados. In J.L. Lanata \& L.A. Borrero (Eds.), Explotación de Recursos Faunísticos en Sistemas Adaptativos Americanos. Arqueología 
Contemporánea, 4, 149-161.

Howey, M. C. L., \& Frederick, K. (2016). Immovable food storage facilities, knowledge, and landscape in nonsedentary societies: Perspectives from northern Michigan. Journal of Anthropological Archaeology, 42, 37-55.

Hyades, P., \& Deniker, J. (2007 [1891]). Los indígenas según Hyades \& Deniker. En D. Legoupil \& A. Prieto (Eds.), Etnografía de los indios Yaghan en la Misión Científica del Cabo de Hornos 1882-1883 (pp. 69-329). Punta Arenas: UMAG-Instituto Francés de Estudios Andinos.

Johnson, L. (1976). Informe sobre una prospección arqueológica en Magallanes (14 diciembre 1976-6 enero 1977). Anales del Instituto de la Patagonia, 7, 87-94.

Keegan, W. F., \& Diamond, J. M. (1987). Colonization of Islands by Islands: A Biogeographical Perspective. Advances in Archaeological Method and Theory, 10, 49-92.

Keegan, W. F., Fitzpatrick, S. M., Sullivan Sealey, K., LeFebure, M. J., \& Sinelli, P.T. (2008). The Role of Small Islands in Marine Subsistence Strategies: Case Studies from the Caribbean. Human Ecology, 36, 635-654.

Kligmann, D. (1992). Reconstrucción de las cadenas operativas de los recursos líticos del sitio Río Pipo 17 (Tierra del Fuego). Tesis de Licenciatura, Universidad de Buenos Aires.

Kligmann, D. (1995). Análisis tipológico y tecno-morfológico del conjunto artefactual lítico del sitio Río Pipo 17, Tierra del Fuego. Arqueología 5, 137-165.

Koppers, W. (1924). Unter Feuerland-Indianern. Verlag Stredcker und Schroder, Stuttgart.

Laming-Emperaire, J. (1972). Los sitios arqueológicos de los archipiélagos de Patagonia Occidental. Anales del Instituto de la Patagonia, 3, 87-96.

Lee, R.B. (1979). The!Kung San: Men, Women and Work in a Foraging Society. Cambridge: Cambridge University Press.

Legoupil, D. (1993-1994). El archipiélago del cabo de Hornos y la costa sur de la isla Navarino: poblamiento y modelos económicos. Anales del Instituto de la Patagonia, 22, 101-121.

Legoupil, D., \& Prieto, A. (1991). Sepultura de niños canoeros en un abrigo pintado en Última Esperanza, Chile. Anales del Instituto de la Patagonia, 20, 133-138.

Legoupil, D., \& Fontugne, M. (1997). El Poblamiento Marítimo en los Archipiélagos de Patagonia: Núcleos Antiguos y Dispersión Reciente. Anales del Instituto de la
Patagonia, 25, 75-87.

Legoupil, D., \& Chevallay, D. (2017). La Terre de Feu (1925): Un documental francés sobre los indígenas de Patagonia y Tierra del Fuego. Magallania, 45(2), 67-80.

Legoupil, D., Prieto, A., \& Sellier, P. (2004). La cueva de los niños (seno Última Esperanza): nuevos hallazgos. Magallania, 32, 225-227.

Lothrop, S. K. (1928). The Indians of Tierra del Fuego. Museum of the American Indian, New York.

Lupo, K. D., \& Schmitt, D. N. (2016). When bigger is not better: The economics of hunting megafauna and its implications for Plio-Pleistocene hunter-gatherers. Journal of Anthropological Archaeology, 44, 185197.

MacArthur, R., \& Wilson, E.O. (1967). The Theory of Island Biogeography. Princeton: Princeton University Press.

Mansur, M. E., \& Lasa, A. (2005). Diversidad artefactual vs. Especialización funcional. Análisis del IV componente de Túnel I (Tierra del Fuego, Argentina). Magallania, 33(2), 69-91.

Mansur, M. E., \& Lasa, A. (2008). Tecnología y función en el IV Componente de Túnel I (Tierra del Fuego). En A. Austral \& M. Tamagnini (Eds.), Problemática de la arqueología contemporánea, II, 297-307. Universidad Nacional de Río Cuarto, Río Cuarto.

Mansur-Franchomme, M. E., Orquera, L. A., \& Piana, E. L. (1987-1988). El alisamiento de la piedra entre cazadores-recolectores: El Caso de Tierra del Fuego. Runa, XVII-XVIII, 111-205.

Martial, L. F. (2005 [1888]). Misión al Cabo de Hornos. Ushuaia: Zagier \& Urruty Publications.

Martial, L. F. (2007 [1891]). Los indígenas según Martial. En D. Legoupil \& A. Prieto (Eds.), Etnografía de los indios Yaghan en la Misión Científica del Cabo de Hornos 1882-1883 (pp. 18-68). Punta Arenas: Ediciones Universidad de Magallanes-Instituto Francés de Estudios Andinos.

Martinic, M. (2011). El occidente fueguino. Todavía una incógnita. Gasco Magallanes, Punta Arenas.

Maximiano Castillejo, A. (2016). From virtual survey to real prospection: Kaw'esqar mobility in the Fuego-Patagonia seascape across terrestrial passages. Quaternary International, 435, 114-127.

Morello, F. (2005). Tecnología y métodos para el desbaste de lascas en el norte de Tierra del Fuego: los núcleos del sitio Cabo San Vicente. Magallania, 33, 29-56.

Morello, F. (2016). L'industrie lithique de part et d'autre du Détroit de Magellan, en Patagonie et Terre de Feu: dynamiques techno-culturelles du peuplement durant 
l'Holocène. Tesis de doctorado, L'Université de Paris I Panthéon-Sorbonne, Paris.

Morello, F. (2018). Informe de sitios arqueológicos en Dientes de Navarino: canteras-talleres en la zona altoandina de isla Navarino. Ms.

Morello, F., Torres, J., Martínez, I., Rodríguez, K., ArroyoKalin, M., French, C., \& San Román, M. (2012). Arqueología de la Punta Santa Ana: reconstrucción de secuencias de ocupación de cazadores-recolectores marinos del estrecho de Magallanes, Patagonia austral, Chile. Magallania, 40(2), 129-149.

Morello, F., Alfonso-Durruty, M., Amorosi, T., Martínez, I., Massone, M., Martin, F., Borrero, L. A., Christensen, M., Torres, J., Sierpe, V., Reyes, O., \& San Román, M. (2018). Junius Bird's collections from sites rockshelter 1, 2 and 3 (Beagle Channel, Patagonia, Chile). Trabajo presentado al 83rd Meeting of the Society for American Archaeology, Washington D.C.

Muñoz, C., Cordero, R., \& Artigas, D. (2016). El sitio Alero Picton 1: Nuevo registro de arte rupestre para los canales fueguinos. Magallania, 44(2), 225-231.

Niemeyer, H. (2013). Recuerdos de una expedición. Carta de Hans Niemeyer al baqueano Reinaldo Catalán de Tierra del Fuego. Documentos inéditos para la historia de Magallanes. Introducción y notas de Samuel García Oteiza. Magallania, 40(2), 221-228.

Ocampo, C., \& Rivas, P. (1996). Inspección arqueológica del área de cobertura del Proyecto Río Cóndor, Suroeste de la Tierra del Fuego. Anales del Instituto de la Patagonia, 24, 125-151.

Ocampo, C., \& Rivas, P. (2000). Nuevos fechados 14C de la costa norte de la isla Navarino, costa sur del canal Beagle, Provincia Antártica Chilena, Región de Magallanes. Anales del Instituto de la Patagonia, 28, 197-204.

Ocampo, C., Rivas, P., \& Aspillaga, E. (2000). Chenque en Bahía Felipe, costa noroccidental de Tierra del Fuego. Anales del Instituto de la Patagonia, 28, 215-223.

Orquera, L. A., \& Piana, E. L. (1993-1994). Lancha Packewaia: Actualización y rectificaciones. Relaciones de la Sociedad Argentina de Antropología, 19, 235-239.

Orquera, L. A., \& Piana, E. L. (1995). Túnel VII en la secuencia arqueológica del canal Beagle: hipótesis y expectativas de los investigadores argentinos. Encuentro en conchales fueguinos. En J. Estévez Escalera \& A. Vila Mitjá (Eds.), Treballs D'Etnoarqueologia, 1, 25-45. Universidad Autónoma de Barcelona, Barcelona.

Orquera, L. A., \& Piana, E. L. (1996). El sitio Shamakush I (Tierra del Fuego, República Argentina). Relaciones de la Sociedad Argentina de Antropología, 21, 215265.

Orquera, L. A., \& Piana, E. L. (1999a). Arqueología de la región del canal Beagle (Tierra del Fuego, República Argentina). Sociedad Argentina de Antropología, Buenos Aires.

Orquera, L. A., \& Piana, E. L. (1999b). La vida material y social de los Yámana. Buenos Aires: EUDEBA.

Orquera, L. A., \& Piana, E. L. (2015). La vida material y social de los Yámana. $2^{\circ}$ ed. Ushuaia: Ediciones Monte Olivia.

Orquera, L. A., Sala, A., Piana, E. L., \& Tapia, A. H. (1977). Lancha Packewaia. Arqueología de los canales fueguinos. Buenos Aires: Huemul.

Orquera, L. A., Piana, E. L., Fiore, D., \& Zangrando, A. F. (2012). 10.000 años de fuegos. Arqueología y etnografía del fin del mundo. Buenos Aires: Editorial Dunken.

Ortiz Troncoso, O. (1972). Una nota sobre un yacimiento arqueológico en el archipiélago del Cabo de Hornos. Anales del Instituto de la Patagonia, 3(1-2), 83-85.

Outes, F. (1905). La edad de la piedra en Patagonia. Buenos Aires: Imprenta de Juan A. Alsina.

Outes, F. (1906). Instrumentos modernos de los onas. Anales del Museo Nacional de Buenos Aires, III (VI), $287-$ 296.

Palacios, C., \& Sierpe, V. (2019). Análisis bioarqueológico de un feto anencefálico del sitio arqueológico cueva de los Niños (Provincia de Última Esperanza, Región de Magallanes, Chile): A 29 años de su hallazgo. Magallania, 47(2), 107-124.

Pallo, C., \& Borrazzo, K. (2016). Miraflores rocks in Tierra del Fuego (southernmost South America): Huntergatherer procurement, optimal pathway analysis and social interaction. Journal of Archaeological Science. Reports, 7, 420-431

Piana, E. L., \& Martinioni, D. R. (1998). Informe sobre un yacimiento arqueológico en el valle de Andorra. Jurisdicción del Parque Nacional Tierra del Fuego (informe técnico). Ushuaia, 1-6.

Piana, E. L., \& Orquera, L. A. (1998). Canoe fuegine: etnografia storica e archeologia. L'essemplare del Museo L'. Pigorini. Bulletino di Paletnologia Italiana, 89, Nuova Serie VII, 397-445.

Piana, E. L., Tessone, A., \& Zangrando, A. (2006). Contextos mortuorios en la región del Canal Beagle: del hallazgo fortuito a la búsqueda sistemática. Magallania, 34(1), 87-101.

Piana, E. L., Vázquez, M., \& Álvarez, M. (2008). Nuevos 
resultados del estudio del sitio Ajej I: un aporte a la variabilidad de estrategias de los canoeros fueguinos. Runa, 29, 101-121.

Ponce, J. F., Borromei, A. M., \& Rabassa, J.O. (2011). Evolución del paisaje y de la vegetación durante el Cenozoico tardío en el extremo sudeste del archipiélago Fueguino y Canal Beagle. En A. F. Zangrando, M. Vázquez \& A. Tessone (Eds.), Los cazadores-recolectores del extremo oriental fueguino. Arqueología de península Mitre e Isla de los Estados (pp. 51-63). Sociedad Argentina de Antropología, Buenos Aires.

Prieto, A., \& Cárdenas, P. (2001). Ajuar de cueros en entierros de canoeros en el archipiélago de Tierra del Fuego. Anales Instituto Patagonia, 29, 183-188.

Prieto, A., Chevallay, D., \& Ovando, D. (2000). Los pasos de indios en Patagonia Austral. En Desde el País de los Gigantes. Perspectivas arqueológicas en Patagonia (pp. 87-94). Jornadas de arqueología de la Patagonia IV. Universidad Nacional de la Patagonia Austral, Río Gallegos.

Ratto, N. (2003). Estrategias de caza y propiedades del registro en la Puna de Chaschuil (Depto Tinogasta, Catamarca, Argentina). Tesis Doctoral, Universidad de Buenos Aires.

Routledge, K. (1919). The Mystery of Easter Island. The story of an Expedition. Londres: Impreso por Hazell, Watson y Viney.

San Román, M. (2016). Stratégies économiques et sociales des chasseurs marins de Patagonie: Archéozoologie des sites anciens du détroit de Magellan et des mers intérieures. Tesis de Doctorado. L'Université de Paris I Panthéon-Sorbonne, Paris.

San Román, M., \& Morello, F. (2001). Canal Maule: nuevos antecedentes sobre prácticas funerarias en el archipiélago fueguino. Anales del Instituto de la Patagonia, 29, 149-161.

San Román, M., Vilicic, D., Lukoviek, E., Sierpe, V., Rodríguez, K., Martin, F., Trejo, V., Christensen, M., Torres, J., \& Morello, F. (2018). Maritime Hunter-Gatherers from Southernmost Patagonia (South America, Chile): Discussing Occupation Intensity and Resource Exploitation Strategies for the central Strait of Magellan during the Late Holocene (2500 BP-XVIII Century). Trabajo presentado al 83rd Annual Meeting of the Society for American Archaeology, Washington D.C.

Santiago, F., \& Vázquez, M. (2009). Dietas promediadas: explorando el registro zooarqueológico supraregional en Tierra del Fuego. Revista del Museo de Antropología, 5, 225-238.
Schiavini, A. (1993). Los lobos marinos como recurso para cazadores recolectores marinos. El caso de Tierra del Fuego. Latin American Antiquity, 4(4), 346-366.

Schindler, H. (1995). Un informe etnográfico sobre los onas del año 1765. Runa, 23, 177-185.

SERNAGEOMIN. (2003). Mapa Geológico de Chile: Versión digital. Servicio Nacional de Geología y Minería CDROM, versión 1.0.

Skottsberg, C. (1913). Observations on the natives of the patagonian channel region. American Anthropologist, $15,578-616$.

Skottsberg, C. (2004 [1919-1911]). La Patagonia salvaje. Ushuaia: Zagier \& Urruty Publications.

Smith, B. (1979). Wind, Waves and Whales. Cape Horn by Kayak. Expedition, 9(4), 10-14.

Solari, M. E. (1992). Anthracologie et ethnoarchéologie dans l'archipel du Cap Horn (Chili). Bulletin Société Botanique Francaise, 139, 407-419.

Speth, J. D. (2010). The Paleoanthropology and Archaeology of Big-Game Hunting. New York: Springer.

Speth, J. D. (2017). Putrid Meat and Fish in the Eurasian Middle and Upper Paleolithic: Are We Missing a Key Part of Neanderthal and Modern Human Diet? PaleoAnthropology, 2017, 44-72.

Speth, J. D. (2018). A New Look at Old Assumptions. Paleoindian Communal Bison Hunting, Mobility and Stone Tool Technology. In K. Carlson \& L.C. Bement (Eds.), The Archaeology of Large-Scale Manipulation of Prey. The Economic and Social Dynamics of Mass Hunting (pp. 161-285). University Press of Colorado, Louisville.

Terradas, X. (2001). La gestión de los recursos minerales en las sociedades cazadoras-recolectoras. Treballs d'etnoarqueologia. Madrid.

Thomas, D. H. (1985). The Archaeology of Hidden Cave. Anthropological Papers of the American Museum of Natural History 61(1), 1-430.

Thomas, D. H. (2015). Engineering Alta Toquima. Social investments and Dividends at 11,000 Feet. En L. L. Scheiber \& M. N. Zedeño (Eds.), Engineering Mountain Landscapes. An Anthropology of Social Investment (pp. 49-74). The University of Utah Press, Salt Lake City.

Tivoli, A., \& Zangrando, A. F. (2011). Subsistence variations and landscape use among maritime hunter-gatherers. A zooarchaeological analysis from the Beagle Channel (Tierra del Fuego, Argentina). Journal of Archaeological Science, 38 (5), 1148-1156.

Torres, J. (2009). La pesca entre los cazadores recolectores 
terrestres de la isla grande de Tierra del Fuego, desde la prehistoria a tiempos etnográficos. Magallania, 37(2), 109-138.

Torres, J. (2013). Cazadores recolectores de Tierra del Fuego y su cercanía al mundo costero: Una aproximación desde la pesca. En J. Oría \& A. Tivoli (Eds.), Cazadores de mar y tierra, estudios recientes en arqueología fueguina (pp. 219-241). Editorial Cultural Tierra del Fuego. Museo del Fin del Mundo, Ushuaia.

Torres, J., \& San Román, M. (2018). Les pêcheries de pierre sur les côtes de Patagonie et de Terre de Feu: la rencontre de traditions techniques aux parcours historiques differents. Book of Abstracts. 18th UISPP World Congress, (pp. 1984-1985), Paris.

Vázquez, M., \& Zangrando, F. (2017). Estructuras de pesca en el canal Beagle. Magallania, 45(1), 101-122.

Vázquez, M., Zangrando, A. F., Tessone, A., \& Ceraso, A. (2011). Arqueología de la costa meridional de Península Mitre. En A.F. Zangrando, M. Vázquez \& A. Tessone (Eds.), Los cazadores-recolectores del extremo oriental fueguino. Arqueología de península Mitre e Isla de los Estados (pp. 203-229). Sociedad Argentina de Antropología, Buenos Aires.

Vega Delgado, C. (1995). Cuando el cielo se oscurece (Samán ankachoé). Historia de vida, testimonio alacalufe de Alberto Achacaz Walakial. Punta Arenas: Editorial Atelí.

Weddell, J. (2006 [1825]). Un viaje hacia el Polo Sur realizado en los años 1822-1824. Buenos Aires: Eudeba.

Yesner, D. (1990). Fuegians and Other Hunter-Gatherers of the Sub-Antarctic Region: 'Cultural Devolution' Reconsidered. In B. Meehan \& N. White (Eds.), HunterGatherer Demography. Past and present. Oceania Monograph, 39, 1-22. University of Sydney, Sydney.

Yesner, D. (2004). Prehistoric Maritime Adaptations of the Subarctic and Subantarctic Zones: The Aleutian/ Fuegian Connection Reconsidered. Arctic Anthropology, 41(2), 76-97.

Zangrando, A. F. (2009). Historia evolutiva y subsistencia de cazadores-recolectores marítimos de Tierra del Fuego. Sociedad Argentina de Antropología, Buenos Aires.

Zangrando, A. F. (2018). Poblamiento temprano y arqueología de costas en Patagonia y Tierra del Fuego: vacío de información, preconceptos y perspectivas. Intersecciones en Antropología, 19, 63-97.

Zangrando, A. F., Pinto, G., \& Tivoli, A. M. (2016). Decreased foraging return in shellfishing? Species composition and shell size of blue mussel (Mytilus edulis) from a Late Holocene site of the South Coast of Tierra del Fuego. Quaternary International, 427, 160-169.

Zangrando, A. F., Vázquez, M., Tessone, A., Tivoli, A. M., Martinoli, M. P., Fernández Ropero, C., Alunni, D., \& Pinto Vargas, G. (2017). Primeros datos sobre la arqueología de bahía Franklin, Isla de los Estados. Trabajo presentado a las X Jornadas de Arqueología de la Patagonia, Puerto Madryn.

Zárraga, C. (2016). Cristina Calderón. Memorias de mi abuela Yagán. Punta Arenas: Ediciones Pix. 